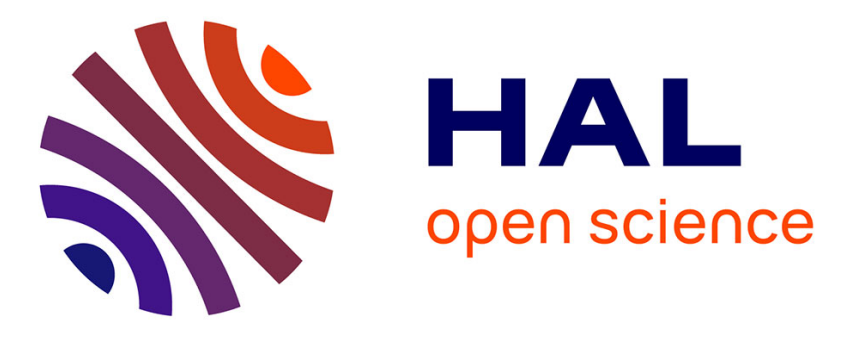

\title{
PI Passivity-Based Control and Performance Analysis of MMC Multi-terminal HVDC Systems
}

\author{
Gilbert Bergna-Diaz, Daniele Zonetti, Santiago Sanchez, Romeo Ortega, \\ Elisabetta Tedeschi
}

\section{- To cite this version:}

Gilbert Bergna-Diaz, Daniele Zonetti, Santiago Sanchez, Romeo Ortega, Elisabetta Tedeschi. PI Passivity-Based Control and Performance Analysis of MMC Multi-terminal HVDC Systems. IEEE Journal of Emerging and Selected Topics in Power Electronics, 2019, 7 (4), pp.2453-2466. 10.1109/JESTPE.2018.2889152 . hal-02419459

\section{HAL Id: hal-02419459 \\ https://hal-centralesupelec.archives-ouvertes.fr/hal-02419459}

Submitted on 17 Jun 2020

HAL is a multi-disciplinary open access archive for the deposit and dissemination of scientific research documents, whether they are published or not. The documents may come from teaching and research institutions in France or abroad, or from public or private research centers.
L'archive ouverte pluridisciplinaire HAL, est destinée au dépôt et à la diffusion de documents scientifiques de niveau recherche, publiés ou non, émanant des établissements d'enseignement et de recherche français ou étrangers, des laboratoires publics ou privés. 


\title{
PI Passivity-based Control and Performance Analysis of MMC Multi-Terminal HVDC Systems
}

\author{
Gilbert Bergna-Diaz, Member IEEE, Daniele Zonetti, Santiago Sanchez, Member IEEE, \\ Romeo Ortega, Fellow IEEE, and Elisabetta Tedeschi, Member IEEE
}

\begin{abstract}
In this work, a decentralized PI passivity-based controller (PI-PBC) is applied to Modular Multilevel Converters (MMCs) to ensure global stability of a multi-terminal HVDC system. For the derivation of the controller, an appropriate model with constant steady-state solutions is obtained via a multifrequency orthogonal coordinates transformation. The control design is next completed using passivity arguments and performance guarantees are established by a small-signal analysis. The obtained results are validated by means of detailed timedomain simulations both on a single-terminal and a four-terminal benchmark.
\end{abstract}

Keywords-Modular Multilevel Converter, PI Passivity-based Control, Port-Hamiltonian Modelling.

\section{INTRODUCTION}

Multi-Terminal (MT) High-Voltage Direct-Current (HVDC) transmission systems form part of one of the major infrastructure developments of our times. This kind of architecture is the preferred solution for the integration of large volumes of renewable energy into the existing power grids, since it potentially mitigates the inherent intermittency of the remotely located and uneven geographically distributed sources by interconnecting them over very long distances [1].

The main components of MT-HVDC grids are ac to dc power converters, which interface ac sources-typically associated to renewable energy source or to ac grids-to a highvoltage dc network through a dc bus. Although two-level voltage source converters (2L-VSCs) have been traditionally employed for this purpose, in recent years we have witnessed the rise in popularity of Modular Multilevel Converters (MMCs) [2]. Compared to 2L-VSCs, MMCs have significant advantages for HVDC applications, especially in terms of reduced losses, modularity, scalability and low harmonic distortion in the output ac voltage with correspondingly reduced requirements for filtering [3].

Independently from the choice of the specific components, MT-HVDC grids are expected to be multi-vendor by nature, thus posing the problem of interoperability of the components, in particular of the power converters and the corresponding local controllers [4]. Although the concept of interoperability has several valid interpretations, it is widely acknowledged that a key role is played in this context by the stability and performance properties of the closed-loop system [5]. The analysis of such properties is far from being trivial, since power converter local controllers are usually implemented by different

Manuscript received June 15, 2018; revised September 23, 2018; accepted November 28, 2018. The work of NTNU was funded by the Norwegian Research Council and DNV-GL through the NFR contract n. 250493-within the "Integrated approach to the Design and Control of Offshore HVDC Networks (IDeCON)" project.

G. Bergna-Diaz, S. Sanchez and E. Tedeschi are with the Department of Electric Power Engineering, Norwegian University of Science and Technology (NTNU), 7491 Trondheim, Norway, (e-mails: gilbert.bergna@ntnu.no, santiago.sanchez@ntnu.no, elisabetta.tedeschi@ntnu.no).

D. Zonetti is with the Laboratoire de Spécification et Vérification, CNRS, ENS Paris-Saclay, 94235 Cachan, France (email:daniele.zonetti@1sv.fr).

R. Ortega is with the Laboratoire des Signaux et Systèmes/CNRS/Supélec, 91190 Gif-sur-Yvette, France (email: romeo.ortega@1ss.supelec.fr). vendors and are subjected to confidentiality agreements, yet they require seamless plug and play operation [1].

A possible approach for a stability and performanceguaranteed control design with the desired plug and play features is based on passivity theory [6]. In fact, the notion of passivity can be used to identify a wide class of stabilizing controllers-see [7]-[9] and reference therein for an overview on this topic-and for which a performance analysis can be realized a posteriori. Nevertheless, motivated by its simplicity, robustness and popularity in industrial applications, the use of PI passivity-based control (PI-PBC) design is largely preferred in practice. Contrary to conventional PI controllers that usually act directly on error signals of interest, the PI-PBC acts instead on the passive output of the system. Interestingly, driving the passive output of the system to zero would ensure global (asymptotic) stability [10], making the PI-PBC a promising control design alternative able to cope with large-signal perturbations. PI-PBC has been already, successfully applied to traditional power converters and MT-HVDC systems based on 2L-VSCs, with the purpose of guaranteeing stability and adequate performance of the closed-loop system [10], [11].

Unfortunately, the application of the same methodology adopted in [11], [12] for the case of MT-HVDC systems based on 2L-VSCs is not straightforward, when these are replaced by MMCs, the reasons being the following. First, conventional coordinate transformations consisting of a single frequency Park transform fail to map the desired steady-state behavior of the system-which consist of periodic solutions in $a b c$ coordinates - to a fixed equilibrium point in $d q z$ coordinates, thus stimying the formulation of the control problem as a regulation problem-refer to [13], [14] for more details. This is since, unlike $2 \mathrm{~L}-\mathrm{VSCs}$, MMCs present multiple frequency components in their steady-state voltage and current variables [13]. Second, the design requires the system to be represented in a suitable port-Hamiltonian form, this being not obvious, considering that MMCs are characterized by complex internal dynamics associated to the circulating currents and the internal capacitor voltages of the upper and lower arms of each phase [15], [16].

To overcome these shortcomings, in this paper we propose an equivalent averaged formulation of an MMC with timeinvariant solutions (along the lines of [13], [14]) and next represent the system using an appropriate port-Hamiltonian representation. This is done in Section II. The modelling of a general HVDC system based on $\mathrm{MMCs}^{1}$ is then developed in Section III, using the same formalism adopted in [11]. Based on such a representation, in Section IV the results of [10], [11] are directly applied both for the case of an individual MMC and of an MT-HVDC with meshed topology. The stability properties and performance guarantees of the resulting controllers are validated and thoroughly analyzed with the support of detailed time-domain simulations on a single and meshed four-terminal configuration in Section V. Thus, this work extends our initial result presented in [17], which was limited to the radial case, and lacked detailed time-

${ }^{1}$ In the remainder of this paper, unless explicitly stated, we refer to an HVDC system based on MMCs simply as an HVDC system. 


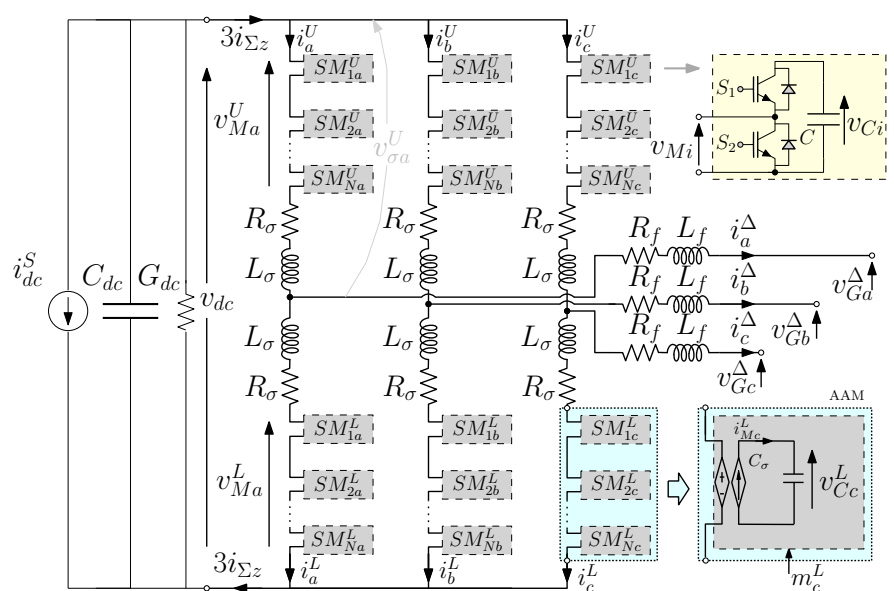

Fig. 1. Topology of the modular multilevel converter under consideration.

domain validation.

Notation. All vectors are column vectors. Given positive integers $n, m$, symbols $\underline{0}_{n} \in \mathbb{R}^{n}$ denotes the vector of all zeros, $\mathbb{1}_{n} \in \mathbb{R}^{n}$ the vector with all ones, $\mathbb{I}_{n}$ the $n \times n$ identity matrix, $\underline{0}_{n \times m}$ the $n \times m$ column matrix of all zeros. $x:=\operatorname{col}\left(x_{1}, \ldots, x_{n}\right) \in \mathbb{R}^{n}$ denotes a vector with entries $x_{i} \in \mathbb{R}$, when clear from the context it is simply referred as $x:=\operatorname{col}\left(x_{i}\right) \cdot \operatorname{diag}\left(a_{i}\right)$ is a diagonal matrix with entries $a_{i} \in \mathbb{R} . \operatorname{bdiag}\left\{A_{i}\right\}$ is a block diagonal matrix with entries $A_{i} \in \mathbb{R}^{n \times n}$. We define the matrix $\mathbb{J}_{2}:=\left[\begin{array}{cc}0 & -1 \\ 1 & 0\end{array}\right] \in \mathbb{R}^{2 \times 2}$. For a function $f: \mathbb{R}^{n} \rightarrow \mathbb{R}, \nabla f$ denotes the transpose of its gradient. We use $\overline{\sin }(\cdot)_{+} \in \mathbb{R}^{3}, \overline{\cos }(\cdot)_{+} \in \mathbb{R}^{3}$ to denote threephase balanced signals in positive sequence. Alternatively we use $\overline{\sin }(\cdot)_{-} \in \mathbb{R}^{3}, \overline{\cos }(\cdot)_{-} \in \mathbb{R}^{3}$ to denote three-phase balanced signals in negative sequence. The symbol $\circ$ denotes the Hadamard product, i.e. the element-wise multiplication between vectors.

\section{MODELING OF MODULAR MULTILEVEL CONVERTERS}

\section{A. Arm Averaged Model}

A single MMC with three upper and three lower arms in half bridge configuration is considered, the topology of which is represented in full detail in Fig. 1. Both upper and lower arms are constituted by an arm inductor-characterized by an inductance $L_{\sigma}$ and a resistance $R_{\sigma}$-connected in series with $N$ sub-modules (SMs). Each SM includes two IGBTs, their respective free wheeling diode and an equivalent capacitor. The circuit is completed by an $R L$ element, located at the ac output, that takes into account the leakage inductance of the transformer and additional inductive filters.

Due to the complexity of the system, suitable, simplified models are usually employed in literature, based on the following, widely accepted, assumptions—see [16], [18]-[21] for more details.

A1. Switching dynamics are neglected.

A2. All SMs voltage signals in the same arm are identical.

A3. Balanced operation of the phases.

Assumption A1 is justified by the very fast operation of the switches, compared to the time-scale at which the other electrical variables evolve. Assumption A2 allows to represent the MMC capacitive dynamics with only one equivalent capacitor in each arm, significantly reducing the size of the model. Indeed, the validity of this assumption rests on a low level SM capacitor balancing algorithm that would ensure equal voltage among the arm SMs [2]. Furthermore, it was proven in [22] that the model derived under this assumption remains accurate even for realistic MMC designs intended for HVDC applications characterized by a large number of levels, which do not typically exhibit strictly equal individual sub-module capacitor voltages per $\mathrm{arm}^{2}$. Finally, Assumption A3 can be legitimized by appropriate design of the system components. Under these assumptions, the switching dynamics of the seriesconnected SMs can be approximated by their averages, which leads to the following average model of the upper $(j=U)$ and lower $(j=L)$ arms:

$$
\begin{aligned}
C_{\sigma} \dot{v}_{C a b c}^{j} & =m_{a b c}^{j} \circ i_{a b c}^{j} \\
L_{\sigma} \dot{i}_{a b c}^{j} & =-R_{\sigma} i_{a b c}^{j}-m_{a b c}^{j} \circ v_{C a b c}^{j}+v_{\sigma}^{j},
\end{aligned}
$$

where we have further defined: as state vector, the collection of the three-phase arm voltage $v_{C a b c}^{j} \in \mathbb{R}^{3}$ and current $i_{a b c}^{j} \in \mathbb{R}^{3}$; as control vector, the three-phase insertion index $m_{a b c}^{j} \in \mathbb{R}^{3}$; as input vector, the three-phase voltage $v_{\sigma}^{j} \in \mathbb{R}^{3}$; as parameters $C_{\sigma}, L_{\sigma}, R_{\sigma} \in \mathbb{R}_{+}$, corresponding respectively to the arm equivalent capacitance, inductance and resistance.

To facilitate the interconnection of the arms dynamics with the $R L$ element preceding the grid, it is convenient to transform the system (II.1) in more suitable $\Sigma-\Delta$ coordinates, which are defined as follows:

$$
\begin{aligned}
v_{C a b c}^{\Sigma} & :=v_{C a b c}^{U}+v_{C a b c}^{L}, & v_{C a b c}^{\Delta}:=v_{C a b c}^{U}-v_{C a b c}^{L} \\
i_{a b c}^{\Sigma}: & :=\frac{1}{2}\left(i_{a b c}^{U}+i_{a b c}^{L}\right), & i_{a b c}^{\Delta}:=i_{a b c}^{U}-i_{a b c}^{L} \\
m_{a b c}^{\Sigma} & :=m_{a b c}^{U}+m_{a b c}^{L}, & m_{a b c}^{\Delta}:=m_{a b c}^{U}-m_{a b c}^{L},
\end{aligned}
$$

where currents $i_{a b c}^{\Sigma}$ and $i_{a b c}^{\Delta}$ have clear physical meanings, since they correspond to the three-phase circulating current and the three-phase current flowing into the grid through the $R L$ element, see also Fig. 1. After some simple manipulations, the arms dynamics in $\Sigma-\Delta$ coordinates can be combined with the dynamics of the $R L$ element, thus leading to:

$$
\begin{aligned}
& C_{\sigma} \dot{v}_{C a b c}^{\Sigma}=m_{a b c}^{\Sigma} \circ i_{a b c}^{\Sigma}+\frac{1}{2} m_{a b c}^{\Delta} \circ i_{a b c}^{\Delta} ; \\
& C_{\sigma} \dot{v}_{C a b c}^{\Delta}=m_{a b c}^{\Delta} \circ i_{a b c}^{\Sigma}+\frac{1}{2} m_{a b c}^{\Sigma} \circ i_{a b c}^{\Delta} ; \\
& L_{\sigma} i_{a b c}^{\Sigma}=\frac{\mathbb{1}_{3} v_{d c}}{2}-R_{\sigma} i_{a b c}^{\Sigma}-\frac{m_{a b c}^{\Sigma} \circ v_{C a b c}^{\Sigma}+m_{a b c}^{\Delta} \circ v_{C a b c}^{\Delta}}{4} ; \\
& L_{\delta} \dot{i}_{a b c}^{\Delta}=-R_{\delta} i_{a b c}^{\Delta}-\frac{m_{a b c}^{\Sigma} \circ v_{C a b c}^{\Delta}+m_{a b c}^{\Delta} \circ v_{C a b c}^{\Sigma}}{4}-v_{G a b c}^{\Delta} ;
\end{aligned}
$$

where we used the following relations-obtained by inspection of Fig. 1:

$$
\begin{aligned}
& v_{\sigma a b c}^{U}+v_{\sigma a b c}^{L}=\mathbb{1}_{3} v_{d c}, \\
& v_{\sigma a b c}^{U}-v_{\sigma a b c}^{L}=-2\left(R_{f} i_{a b c}^{\Delta}+L_{f} \dot{i}_{a b c}^{\Delta}+v_{G a b c}^{\Delta}\right)
\end{aligned}
$$

and further defined the equivalent ac inductance $L_{\delta}:=L_{\sigma} / 2+$ $L_{f}$ and resistance $R_{\delta}:=R_{\sigma} / 2+R_{f}$. The model (II.2) is referred in the sequel as the Arm Averaged Model (AAM) of the MMC.

\section{B. Steady-state analysis}

A fundamental step towards the analysis and the control design is the identification of the steady-states of interest for the correct and safe operation of an MMC. It is typically required that - see [2], [15] for a precise justification:

\section{R1. The ac grid current is a periodic three-phase sinusoidal}

${ }^{2}$ Low-level arm balancing algorithms with reduced switching frequency in the lines of [23] are usually used to decrease the switching losses in HVDC applications, which result in having some differences in the SMs capacitor voltages within the same arm. 
signal at the fundamental frequency $\omega>0$, i.e.

$$
i_{\mathrm{ss}}^{\Delta}=I_{\mathrm{ss}}^{\Delta} \overline{\sin }\left(\omega t+\varphi_{\mathrm{ss}}^{\Delta}\right)_{+}, \quad I_{\mathrm{ss}}^{\Delta} \in \mathbb{R}, \varphi_{\mathrm{ss}}^{\Delta} \in \mathbb{S}
$$

R2. The upper arm insertion indices are phase-shifted of $\pi \mathrm{rad}$ with respect to the lower arm insertion indices, i.e.

$$
m_{\mathrm{ss}}^{\Sigma} \approx \mathbb{1}_{3}, \quad m_{\mathrm{ss}}^{\Delta} \approx M_{\mathrm{ss}} \overline{\sin }(\omega t)_{+} ;
$$

R3. The sum of the circulating current in all phases is a dc signal, i.e.

$$
\mathbb{1}_{3}^{\top} i_{\mathrm{ss}}^{\Sigma}=\text { const }
$$

R4. The sum of arm capacitor voltages is a dc signal, i.e.

$$
\mathbb{1}_{3}^{\top} v_{C, \mathrm{ss}}^{\Sigma}=\text { const. }
$$

These requirements complicate the control design, since the steady-state of interest does not coincide with a fixed equilibrium point. However, an analysis of the steady-state oscillations exhibited by (II.2) in simulations revealed the following situation in steady-state conditions [13]:

- $\Delta$-variables consist mainly of the sum of a signal oscillating at the fundamental frequency $\omega$ with a signal oscillating at frequency $3 \omega$.

- $\Sigma$-variables consist mainly of the sum of a de signal with a signal oscillating at frequency $-2 \omega$.

Remark 1. Actually, $\Sigma$ - and $\Delta$-variables are in general characterized at steady-state by additional higher order harmonics. However, their magnitude is small and therefore can be neglected [13].

Remark 2. Note that, at steady-state, the grid current $i^{\Delta}$ only oscillates at the fundamental frequency by definition, while the circulating current $i^{\Sigma}$ might consists of a simple dc signal, in the case that a Circulating Current Suppression Controller is adopted [24].

\section{A multi-frequency coordinates transformation}

The classification presented in the previous section was instrumental in [13], [14] to determine a suitable, multifrequency coordinates transformation that maps the oscillating steady-states of interest to constant quantities, while preserving the original model nonlinear structure and avoiding the dynamic phasor approximation used in [25]. This approach, based on an appropriate combination of Park and rotational transformations, is sketched here for the sake of completeness.

We first introduce the following definitions. A positive and negative sequence Park transformation of a three-phase signal $x=\operatorname{col}\left(x_{a}, x_{b}, x_{c}\right) \in \mathbb{R}^{3}$ with transformation angle $\phi(t)=$ $\omega_{0} t \in \mathbb{R}, \omega_{0}>0$, are respectively defined by:

$$
\begin{aligned}
x_{d q z} & :=\frac{2}{3}\left[\begin{array}{c}
\overline{\cos }\left(\omega_{0} t\right)_{+}^{\top} \\
\sin \left(\omega_{0} t\right)_{+}^{\top} \\
\frac{1}{2} \mathbb{1}_{3}^{\top}
\end{array}\right]\left[\begin{array}{l}
x_{a} \\
x_{b} \\
x_{c}
\end{array}\right]=P_{\omega_{0}} x_{a b c}, \\
x_{d q z} & :=\frac{2}{3}\left[\begin{array}{c}
\frac{\cos \left(\omega_{0} t\right)_{-}^{\top}}{\sin \left(\omega_{0}\right) t_{-}^{\top}} \\
\frac{1}{2} \mathbb{1}_{3}^{\top}
\end{array}\right]\left[\begin{array}{l}
x_{a} \\
x_{b} \\
x_{c}
\end{array}\right]=P_{-\omega_{0}} x_{a b c},
\end{aligned}
$$

with $x_{d q z}=\operatorname{col}\left(x_{d}, x_{q}, x_{z}\right) \in \mathbb{R}^{3}$. Let $x_{a b c}^{\Delta} \in \mathbb{R}^{3}, x_{a b c}^{\Sigma} \in \mathbb{R}^{3}$, be general three-phase signals in $\Delta$-, $\Sigma$-coordinates respectively, and define the following transformations:

$$
\begin{aligned}
& x_{d q z}^{\Sigma}:=\operatorname{col}\left(x_{d q}^{\Sigma}, x_{z}^{\Sigma}\right)=P_{-2 \omega} x_{a b c}^{\Sigma}, \\
& x_{d q z}^{\Delta}:=\operatorname{col}\left(x_{d q}^{\Delta}, x_{z}^{\Delta}\right)=P_{\omega} x_{a b c}^{\Delta} .
\end{aligned}
$$

By splitting $d q$ and zero components, the AAM model (II.2) in new coordinates—-see [13] for a detailed derivation—reads then:

$$
\begin{aligned}
C_{\sigma} \dot{v}_{C d q}^{\Sigma} & =i_{M d q}^{\Sigma}+\mathbb{J}_{2} C_{\sigma} 2 \omega v_{C d q}^{\Sigma}, \quad C_{\sigma} \dot{v}_{C z}^{\Sigma}=i_{M z}^{\Sigma}, \\
C_{\sigma} \dot{v}_{C d q}^{\Delta} & =i_{M d q}^{\Delta}+\mathbb{J}_{2} C_{\sigma} \omega v v_{C d q}^{\Delta}, \quad C_{\sigma} \dot{v}_{C z}^{\Delta}=i_{M z}^{\Delta}, \\
L_{\sigma} \dot{i}_{d q}^{\Sigma} & =-v_{M d q}^{\Sigma}+\left(\mathbb{J}_{2} L_{\sigma} 2 \omega-R_{\sigma} \mathbb{I}_{2}\right) i_{d q}^{\Sigma}, \\
L_{\sigma} i_{z}^{\Sigma} & =-v_{M z}^{\Sigma}-R_{\sigma} i_{z}^{\Sigma}+\frac{1}{2} v_{d c}, \\
L_{\delta} \dot{i}_{d q}^{\Delta} & =v_{M d q}^{\Delta}+\left(\mathbb{J}_{2} L_{\delta} \omega-R_{\delta} \mathbb{I}_{2}\right) i_{d q}^{\Delta}-v_{G d q}^{\Delta},
\end{aligned}
$$

and the following definitions:

$$
\begin{aligned}
& i_{M}^{\Sigma}:=P_{-2 \omega}\left(P_{-2 \omega}^{-1} m_{d q z}^{\Sigma} \circ P_{-2 \omega}^{-1} i_{d q z}^{\Sigma}+P_{\omega}^{-1} m_{d q z}^{\Delta} \circ \frac{P_{\omega}^{-1} i_{d q z}^{\Delta}}{2}\right) \\
& i_{M}^{\Delta}:=P_{\omega}\left(P_{\omega}^{-1} m_{d q z}^{\Delta} \circ P_{-2 \omega}^{-1} i_{d q z}^{\Sigma}+P_{-2 \omega}^{-1} m_{d q z}^{\Sigma} \circ \frac{P_{\omega}^{-1} i_{d q z}^{\Delta}}{2}\right) \\
& v_{M}^{\Sigma}:=P_{-2 \omega} \frac{P_{-2 \omega}^{-1} m_{d q z}^{\Sigma} \circ P_{-2 \omega}^{-1} v_{C d q z}^{\Sigma}+P_{\omega}^{-1} m_{d q z}^{\Delta} \circ P_{\omega}^{-1} v_{C d q z}^{\Delta}}{4} \\
& v_{M}^{\Delta}:=-P_{\omega} \frac{P_{\omega}^{-1} m_{d q z}^{\Delta} \circ P_{-2 \omega}^{-1} v_{C d q z}^{\Sigma}+P_{-2 \omega}^{-1} m_{d q z}^{\Sigma} \circ P_{\omega}^{-1} v_{C d q z}^{\Delta}}{4}
\end{aligned}
$$

with $i_{M}^{\Sigma}:=\operatorname{col}\left(i_{M d q}^{\Sigma}, i_{M z}^{\Sigma}\right), i_{M}^{\Delta}:=\operatorname{col}\left(i_{M d q}^{\Delta}, i_{M z}^{\Delta}\right)$ and $v_{M}^{\Sigma}:=$ $\operatorname{col}\left(v_{M d q}^{\Sigma}, v_{M z}^{\Sigma}\right), v_{M}^{\Delta}:=\operatorname{col}\left(v_{M d q}^{\Delta}, v_{M z}^{\Delta}\right)$ corresponding to the three-phase modulated currents and voltages respectively.

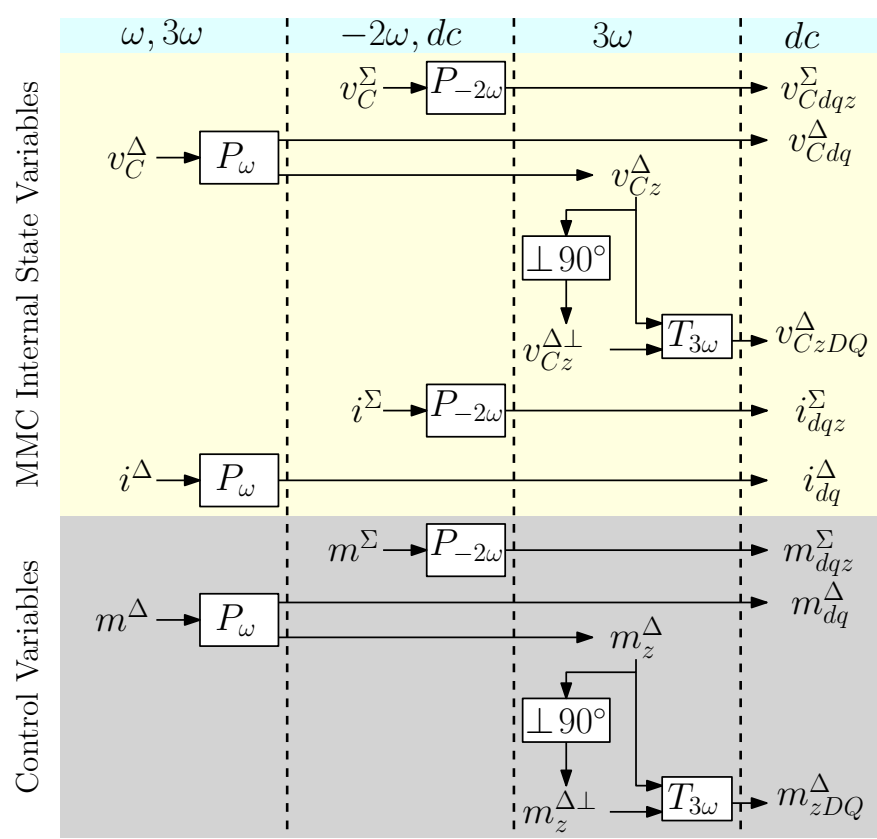

Fig. 2. Multi-frequency coordinates transformation.

Under the aforementioned assumption of negligible highorder harmonics, we have then that the steady-states of the $\Sigma$ variables are mapped to a constant vector $x_{\mathrm{ss}, d q z}^{\Sigma}$. By contrast, $\Delta$-variables are mapped to a vector

$$
x_{\mathrm{ss}, d q z}^{\Delta}=\operatorname{col}\left(x_{d q, \mathrm{ss}}^{\Delta}, x_{z, \mathrm{ss}}^{\Delta}\right) \in \mathbb{R}^{3},
$$

where only $x_{d q, s s}^{\Delta}$ is a constant quantity, while the zerocomponent $x_{z, s s}^{\Delta}$ still oscillates at frequency $3 \omega$. Hence, as illustrated in Fig. 2, the transformation is completed by an additional change of coordinates, which is employed to map these harmonics to constant values. This change of coordinates is usually adopted in single-phase ac systems [26] and employs an auxiliary, virtual variable $x^{\perp} \in \mathbb{R}$, shifted of $\frac{\pi}{2} \mathrm{rad}$ from the original signal $x \in \mathbb{R}$ :

$$
x_{D Q}:=\left[\begin{array}{rr}
\cos \left(\omega_{0} t\right) & \sin \left(\omega_{0} t\right) \\
\sin \left(\omega_{0} t\right) & -\cos \left(\omega_{0} t\right)
\end{array}\right]\left[\begin{array}{l}
x \\
x^{\perp}
\end{array}\right]=T_{\omega_{0}} x,
$$

with $x_{D Q}=\operatorname{col}\left(x_{D}, x_{Q}\right) \in \mathbb{R}^{2}$. Then, by applying this change 
of coordinates to the zero component of $\Delta$-variables we have

$$
x_{z D Q}^{\Delta}=\left[\begin{array}{c}
x_{z D}^{\Delta} \\
x_{z Q}^{\Delta}
\end{array}\right]=T_{3 \omega}\left[\begin{array}{l}
x_{z}^{\Delta} \\
x_{z}^{\Delta \perp}
\end{array}\right],
$$

that, for the only $\Delta$-variables equipped with a zero component, requires the definition of the additional variables

$$
v_{C z D Q}^{\Delta}:=\left[\begin{array}{c}
v_{C z D}^{\Delta} \\
v_{C z Q}^{\Delta}
\end{array}\right], \quad m_{z D Q}^{\Delta}:=\left[\begin{array}{c}
m_{z D}^{\Delta} \\
m_{z Q}^{\Delta}
\end{array}\right] .
$$

\section{Stationary AAM in port-Hamiltonian form}

Before presenting the AAM with time invariant solutions based on the transformations (II.4)-(II.6), an additional yet simple variables and parameters change is adopted. This slight modification eases the derivation of a port-Hamiltonian representation of the system, which is instrumental for the control design carried out in the next section. We then introduce the variables

$$
\hat{m}_{z}^{\Sigma}:=2 m_{z}^{\Sigma}, \quad \hat{v}_{C z}^{\Sigma}:=2 v_{C z}^{\Sigma}, \quad \hat{i}_{z}^{\Sigma}:=2 i_{z}^{\Sigma}, \quad \hat{i}_{d q}^{\Delta}:=i_{d q}^{\Delta} / 2
$$

and the reparametrization:

\begin{tabular}{|c|c|c|c|}
\hline$\hat{L}_{\delta}:=16 L_{\delta}$ & $\hat{L}_{\sigma}:=8 L_{\sigma}$ & $\hat{L}_{\sigma z}:=4 L_{\sigma}$ & \\
\hline$\hat{R}_{\delta}:=16 R_{\delta}$ & $\hat{R}_{\sigma}:=8 R_{\sigma}$ & $\hat{R}_{\sigma z}:=4 R_{\sigma}$ & $\hat{G}_{d c}:=8 G_{d c} / 3$ \\
\hline & $\hat{C}_{\sigma}:=2 C_{\sigma}$ & $\hat{C}_{\sigma z}:=C_{\sigma}$ & $\hat{C}_{d c}:=8 C_{d c} / 3$ \\
\hline
\end{tabular}

Based on these definitions, the AAM model can be rewritten in compact form as

$$
P \dot{z}=A(m) z+E,
$$

with: state vector

$$
z:=\operatorname{col}\left(v_{C d q}^{\Sigma}, \hat{v}_{C z}^{\Sigma}, v_{C d q}^{\Delta}, v_{C z D Q}^{\Delta}, i_{d q}^{\Sigma}, \hat{i}_{z}^{\Sigma}, \hat{i}_{d q}^{\Delta}, v_{d c}\right) \in \mathbb{R}^{13} ;
$$

control vector

$$
m:=\operatorname{col}\left(m_{d q}^{\Sigma}, \hat{m}_{z}^{\Sigma}, m_{d q}^{\Delta}, m_{z D Q}^{\Delta}\right) \in \mathbb{R}^{7}
$$

constant input vector

$$
E:=\operatorname{col}\left(0_{2}, 0,0_{2}, 0_{2}, 0_{2}, 0,-8 v_{G d q}^{\Delta},-\frac{8}{3} i_{d c}\right) \in \mathbb{R}^{13}
$$

matrices $A(m), P \in \mathbb{R}^{13 \times 13}$, with

$$
P=\operatorname{bdiag}\left\{\hat{C}_{\sigma} \mathbb{I}_{2}, \hat{C}_{\sigma z}, \hat{C}_{\sigma} \mathbb{I}_{4}, \hat{L}_{\sigma} \mathbb{I}_{2}, \hat{L}_{\sigma z}, \hat{L}_{\delta} \mathbb{I}_{2}, \hat{C}_{d c}\right\}
$$

and $A(m)$ defined as in (II.8). The model (II.7) will be referred in the sequel as the Stationary Arm Averaged Model (sAAM) of an MMC. Now it can be easily seen that with the use of the energy variables as the new state vector

$$
x=P z \in \mathbb{R}^{13},
$$

containing the collection of charges of the capacitors and the magnetic fluxes of the inductors, i.e.:

$x:=\operatorname{col}\left(q_{C d q}^{\Sigma}, \hat{q}_{C z}^{\Sigma}, q_{C d q}^{\Delta}, q_{C z D Q}^{\Delta}, \phi_{C d q}^{\Sigma}, \hat{\phi}_{C z}^{\Sigma}, \hat{\phi}_{d q}^{\Delta}, q_{d c}\right) \in \mathbb{R}^{13}$,

the system (II.7) can be rewritten in the port-Hamiltonian representation, as

$$
\begin{aligned}
\dot{x} & =(\mathcal{J}(m)-\mathcal{R}) \nabla \mathcal{H}+B+G u_{p}, \quad \mathcal{H}(x):=\frac{1}{2} x^{\top} Q x, \\
y_{p} & =G^{\top} \nabla \mathcal{H}
\end{aligned}
$$

where we have further introduced: the appropriate decomposition of the matrix $A(m)$ in a symmetric positive part $\mathcal{R}=\mathcal{R}^{\top} \geq 0$ referred to as the system dissipation matrix, and a skew-symmetric part $\mathcal{J}=-\mathcal{J}^{\top}$, referred to as the interconnection matrix; the system Hamiltonian $\mathcal{H} \in \mathbb{R}$, with
$Q=P^{-1}$; the decomposition of the vector $E=B+G u_{p}$, with $u_{p}=-i_{d c} \in \mathbb{R}, y_{p}:=\frac{8}{3} v_{d c} \in \mathbb{R}$ and $B, G \in \mathbb{R}^{n}$ defined in the Appendix. In addition, noting that the control variables appear linearly in (II.8), it is possible to write

$$
\mathcal{J}(m):=\mathcal{J}_{0}+\sum_{h=1}^{7} \mathcal{J}_{h} m_{h}
$$

for some appropriate skew-symmetric matrices $\mathcal{J}_{0}, \mathcal{J}_{h} \in \mathbb{R}^{13 \times 13}$, which are given in the Appendix.

Remark 3. Notice that the port-Hamiltonian representation of the MMC in (II.9) emphasizes the role of the energy or Hamiltonian function $\mathcal{H}(x)$ in the system dynamics, as well as the systems interconnection pattern $\mathcal{J}$ and its dissipation $\mathcal{R}$; the main ingredients for passivity-based controllers.

\section{MODELING OF MT-HVDC SYSTEMS}

Following the methodology adopted in [11], a general multi-terminal HVDC system can be viewed as a graph $\mathscr{G}(\mathscr{V}, \mathscr{E}, \mathscr{M})$, where: $\mathscr{V}$ is a set of nodes, with cardinality c, associated to the points of connection between each of the MMCs and the HVDC grid; $\mathscr{E}$ is a set of edges, with cardinality $\mathrm{t}$, associated to transmission lines dynamics; $\mathscr{M} \in \mathbb{R}^{\mathrm{c} \times \mathrm{t}}$ is the incidence matrix of the graph, capturing the HVDC network topology [27].

The HVDC network can be characterized by $t$ meshely connected $R L$ transmission lines, the dynamics of which is described by the following port-Hamiltonian system

$$
\begin{aligned}
& \dot{x}_{\mathrm{t}}=-\mathcal{R}_{\mathrm{t}} \nabla \mathcal{H}_{\mathrm{t}}\left(x_{\mathrm{t}}\right)+\mathscr{M}^{\top} u_{\mathrm{o}} \\
& y_{\mathrm{o}}=\mathscr{M} \nabla \mathcal{H}_{\mathrm{t}}\left(x_{\mathrm{t}}\right)
\end{aligned}
$$

with: state vector $x_{\mathrm{t}}:=\psi_{\mathrm{t}}=\operatorname{col}\left(\psi_{\mathrm{t}, i}\right) \in \mathbb{R}^{\mathrm{t}}$, the collection of the lines magnetic fluxes; interconnection variables $u_{\mathrm{o}}:=\operatorname{col}\left(v_{\mathrm{o}, i}\right) \in \mathbb{R}^{\mathrm{c}}, y_{\mathrm{o}}:=i_{\mathrm{o}, i} \in \mathbb{R}^{\mathrm{t}}$ corresponding respectively to the node voltages and leaving currents; dissipation matrix $\mathcal{R}_{\mathrm{t}}:=\operatorname{bdiag}\left\{\mathcal{R}_{\mathrm{t}, i}\right\} \in \mathbb{R}^{\mathrm{t} \times \mathrm{t}}$, where $R_{\mathrm{t}, i}$ are the lines resistance; energy function

$$
\mathcal{H}_{\mathrm{t}}\left(x_{\mathrm{t}}\right):=\frac{1}{2} x_{\mathrm{t}} Q_{\mathrm{t}} x_{\mathrm{t}}, \quad Q_{\mathrm{t}}:=\operatorname{diag}\left(1 / L_{\mathrm{t}, i}\right),
$$

where $L_{\mathrm{t}, i}$ are the line inductances.

Concerning the MMCs composing the HVDC system, we employ the port-Hamiltonian representation (II.9) that has been already identified in the previous section. Moreover, to provide a compact representation of the $c$ MMCs constituting the HVDC system, we define the aggregated model

$$
\begin{aligned}
\dot{x}_{\mathrm{c}} & =\left[\mathcal{J}_{\mathrm{c}}\left(m_{\mathrm{c}}\right)-\mathcal{R}_{\mathrm{c}}\right] \nabla \mathcal{H}_{\mathrm{c}}+B_{\mathrm{c}}+G_{\mathrm{c}} u_{\mathrm{o}} \\
-y_{\mathrm{o}} & =G_{\mathrm{c}}^{\top} \nabla \mathcal{H}_{\mathrm{c}}
\end{aligned}
$$

with: state vector $x_{\mathrm{c}}:=\operatorname{col}\left(x_{i}\right) \in \mathbb{R}^{\mathrm{cn}}$; interconnection variables $u_{\mathrm{c}}:=v \operatorname{col}\left(v_{\mathrm{c}, i}\right) \in \mathbb{R}^{\mathrm{c}},-y_{\mathrm{c}}:=i_{\mathrm{dc}, i} \in \mathbb{R}^{\mathrm{c}}$ corresponding to the node voltages and entering currents; interconnection, dissipation matrices

$$
\mathcal{J}_{\mathrm{c}}:=\operatorname{bdiag}\left\{\mathcal{J}_{i}\right\} \in \mathbb{R}^{n c \times n c}, \mathcal{R}_{\mathrm{c}}:=\operatorname{bdiag}\left\{\mathcal{R}_{i}\right\} \in \mathbb{R}^{n \mathrm{c} \times n \mathrm{c}} ;
$$

source vector and input matrix

$$
B_{\mathrm{c}}:=\operatorname{col}\left(B_{i}\right) \in \mathbb{R}^{n \mathrm{c}}, G_{\mathrm{c}}:=\operatorname{bdiag}\left\{G_{i}\right\} \in \mathbb{R}^{n \mathrm{c} \times \mathrm{c}},
$$

and the energy function $\mathcal{H}_{\mathrm{c}}:=\sum_{i=1}^{\mathrm{c}} \mathcal{H}_{i}\left(x_{i}\right)$.

Finally, for the interconnection of the MMCs with the HVDC networks, it suffices to combine (III.1) with (III.2) to get the overall model with time-invariant solutions:

$$
\dot{x}_{\text {tot }}=\left(\mathcal{J}_{\text {tot }}\left(m_{\mathrm{c}}\right)-\mathcal{R}_{\text {tot }}\right) \nabla \mathcal{H}_{\text {tot }}+E_{\text {tot }}
$$

with: state vector $x_{\text {tot }}:=\operatorname{col}\left(x_{\mathrm{c}}, \frac{8}{3} x_{\mathrm{t}}\right) \in \mathbb{R}^{n \mathrm{c}+\mathrm{t}}$; interconnec- 
$A(m):=\ldots$

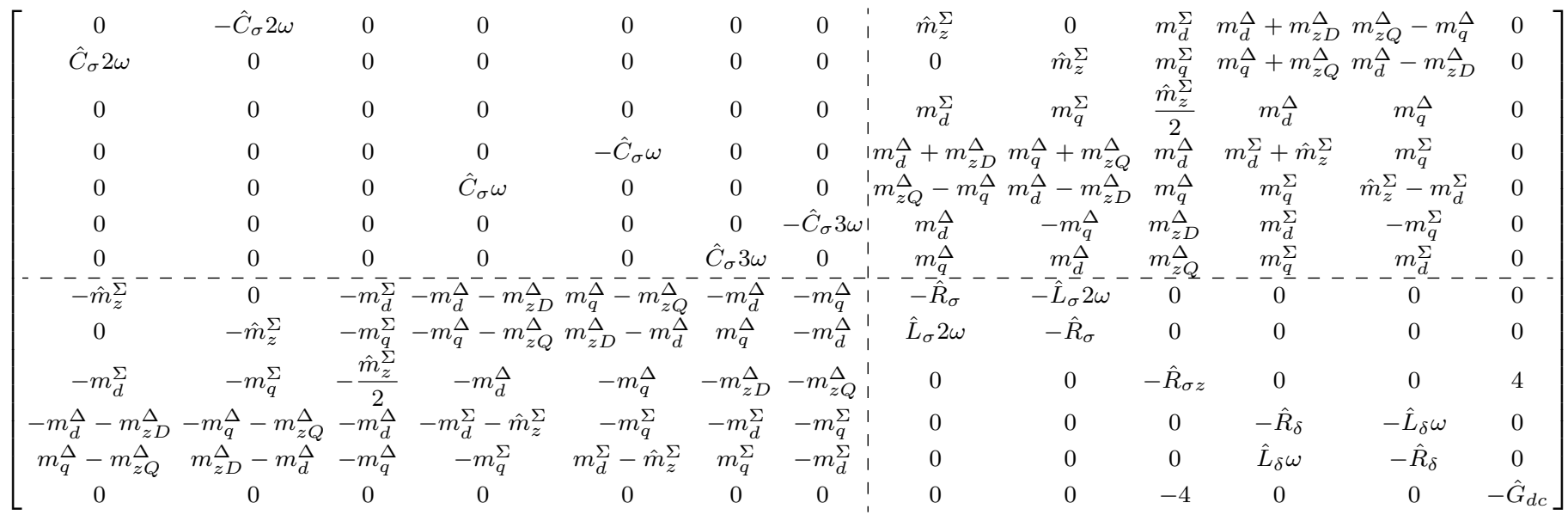

tion, dissipation matrices

$$
\begin{gathered}
\mathcal{J}_{\text {tot }}\left(m_{\mathrm{c}}\right):=\left[\begin{array}{cc}
\mathcal{J}_{\mathrm{c}}\left(m_{\mathrm{c}}\right) & -G_{\mathrm{c}} \mathscr{M} \\
\mathscr{M}^{\top} G_{\mathrm{c}}^{\top} & \underline{0}_{\mathrm{t} \times \mathrm{t}}
\end{array}\right] \in \mathbb{R}^{(n \mathrm{c}+\mathrm{t}) \times(n \mathrm{c}+\mathrm{t})}, \\
\mathcal{R}_{\text {tot }}:=\operatorname{bdiag}\left\{\mathcal{R}_{\mathrm{c}}, \frac{8}{3} \mathcal{R}_{\text {net }}\right\} \in \mathbb{R}^{(n \mathrm{c}+\mathrm{t}) \times(n \mathrm{c}+\mathrm{t})} ;
\end{gathered}
$$

source vector $E_{\text {tot }}:=\operatorname{col}\left(E_{\mathrm{c}}, \underline{0}_{\mathrm{t}}\right) \in \mathbb{R}^{n \mathrm{c}+\mathrm{t}}$, and energy function $\mathcal{H}_{\text {tot }}=\mathcal{H}_{\mathrm{c}}+\frac{3}{8} \mathcal{H}_{\mathrm{t}}$, where the scaling factor $8 / 3$ has been introduced in the HVDC network dynamics subsystem to preserve skew-symmetry.

\section{PI-PASSIVITY BASED CONTROL}

The control methodology follows the results presented in [10] - applicable to averaged models of switched power converters with steady-state time-invariant solutions-and next extended to MT-HVDC systems based on 2L-VSCs in [11]. In both cases, the system was represented in the following port-Hamiltonian form:

$$
\dot{x}=\left(\mathcal{J}_{0}+\sum_{h=1}^{p} \mathcal{J}_{h} m_{h}-\mathcal{R}\right) \nabla \mathcal{H}(x)+E,
$$

where: $x \in \mathbb{R}^{n}$ is the state vector; $m:=\operatorname{col}\left(m_{h}\right) \in \mathbb{R}^{p}$ is the control vector; $E \in \mathbb{R}^{n}$ is the input vector; $\mathcal{J}_{i}=-\mathcal{J}_{i}^{\top}$ and $\mathcal{R}=\mathcal{R}^{\top} \geq 0$ are the $n$-dimensional square interconnection and dissipation matrices; energy function

$$
\mathcal{H}(x)=\frac{1}{2} x^{\top} \operatorname{diag}\left(p_{i}\right) x
$$

for some $p_{i}>0$. Before recalling the main result, the following definition of assignable steady-state is necessary.

Definition IV.1 An admissible steady-state for the system (IV.1) is given by any point $x^{\star} \in \mathbb{R}^{n}$ that verifies:

$$
\underline{0}=\left(\mathcal{J}_{0}+\sum_{h=1}^{p} \mathcal{J}_{h} m_{h}^{\star}-\mathcal{R}\right) \nabla \mathcal{H}\left(x^{\star}\right)+E,
$$

for some constant $m^{\star}:=\operatorname{col}\left(m_{h}^{\star}\right) \in \mathbb{R}^{p}$.

Notice that, since $p<n$ there might exist $x \in \mathbb{R}^{n}$ that do not verify (IV.2) for any $u \in \mathbb{R}^{p}$. We are now ready to recall the main result presented in [10].

Proposition IV.2 Consider a port-Hamiltonian system of the form (IV.1). For any admissible steady-state $x^{\star} \in \mathbb{R}^{n}$, the PI-

\section{PBC controller}

$$
\begin{aligned}
m_{h} & =-K_{P h} y_{h}+K_{I h} \gamma_{h}, \quad \dot{\gamma}_{h}=-y_{h}, \\
y_{h} & =\nabla \mathcal{H}^{\top}\left(x^{\star}\right) \mathcal{J}_{h}^{\top} \nabla \mathcal{H}(x), \quad h=1 \ldots m,
\end{aligned}
$$

with $K_{P}, K_{I} \in \mathbb{R}_{+}^{p \times p}$, guarantees that for all initial conditions $(x(0), \xi(0)) \in \mathbb{R}^{n} \times \mathbb{R}^{m}$ the trajectories of the closed loop system are bounded and $\lim _{t \rightarrow \infty} x(t)=x_{\star}$ if $y$ is detectable.

The previous result is established proving that $y$ is a passive output for the system (IV.1). Hence, since a PI controller is output strictly passive, the closed-loop system is $L_{2}$ stable. See [10] for further details, and [8], [9] for some basic material in passivity theory. Furthermore, it was proven in [11] that a strong connection exists between the passive output and Akagi's instantaneous active and reactive power definitions method for the case of the $2 \mathrm{~L}-\mathrm{VSC}$, as driving $y$ to zero could be reinterpreted as a (active and reactive) power equalization objective.

Since the model of the MMC in single-terminal configuration (II.9) matches with the port-Hamiltonian system (IV.1), the same result reported in [10], resumed in Proposition IV.2, can be applied. In particular, the passive output can be computed according to (IV.3), thus leading to

$$
\begin{aligned}
y_{1}= & \hat{i}_{z}^{\Sigma \star} v_{C d}^{\Sigma}-\hat{i}_{z}^{\Sigma} v_{C d}^{\Sigma \star}+i_{d}^{\Sigma \star} \hat{v}_{C z}^{\Sigma}-i_{d}^{\Sigma} \hat{v}_{C z}^{\Sigma \star}+i_{d}^{\Delta \star}\left(v_{C d}^{\Delta}+v_{C z D}^{\Delta}\right) \\
& -\left(v_{C d}^{\Delta \star}+v_{C z D}^{\Delta \star}\right) i_{d}^{\Delta}+i_{q}^{\Delta \star}\left(v_{C z Q}^{\Delta}-v_{C q}^{\Delta}\right) \\
& -\left(v_{C z Q}^{\Delta \star}-v_{C q}^{\Delta \star}\right) i_{q}^{\Delta} \\
y_{2}= & \hat{i}_{z}^{\Sigma \star} v_{C q}^{\Sigma}-v_{C q}^{\Sigma \star} \hat{i}_{z}^{\Sigma}+i_{q}^{\Sigma \star} \hat{v}_{C z}^{\Sigma}-\hat{v}_{C z}^{\Sigma \star} i_{q}^{\Sigma}+i_{q}^{\Delta \star}\left(v_{C d}^{\Delta}-v_{C z D}^{\Delta}\right) \\
& -\left(v_{C \star}^{\Delta \star}-v_{C z D}^{\Delta \star}\right) i_{q}^{\Delta}+i_{d}^{\Delta \star}\left(v_{C q}^{\Delta}+v_{C z Q}^{\Delta}\right) \\
& -\left(v_{C q}^{\Delta \star}+v_{C z Q}^{\Delta \star}\right) i_{d}^{\Delta} \\
y_{3}= & i_{d}^{\Sigma \star} v_{C d}^{\Sigma}-v_{C d}^{\Sigma \star} i_{d}^{\Sigma}+i_{q}^{\Sigma \star} v_{C q}^{\Sigma}-v_{C q}^{\Sigma \star} i_{q}^{\Sigma}+i_{d}^{\Delta \star} v_{C d}^{\Delta}-v_{C d}^{\Delta \star} i_{d}^{\Delta} \\
& +\frac{1}{2}\left(\hat{i}_{z}^{\Sigma \star} \hat{v}_{C z}^{\Sigma}-\hat{v}_{C z}^{\Sigma \star} \hat{i}_{z}^{\Sigma}\right)+i_{q}^{\Delta \star} v_{C q}^{\Delta}-v_{C q}^{\Delta \star} i_{q}^{\Delta}, \\
y_{4}= & i_{d}^{\Delta \star}\left(v_{C d}^{\Sigma}+\hat{v}_{C z}^{\Sigma}\right)-\left(v_{C d}^{\Sigma \star}+\hat{v}_{C z}^{\Sigma \star}\right) i_{d}^{\Delta}+i_{q}^{\Delta \star} v_{C q}^{\Sigma}-v_{C q}^{\Sigma \star} i_{q}^{\Delta} \\
& +i_{d}^{\Sigma \star}\left(v_{C d}^{\Delta}+v_{C z D}^{\Delta}\right)-\left(v_{C d}^{\Delta \star}+v_{C d}^{\Delta \star}\right) i_{d}^{\Sigma}+\hat{i}_{z}^{\Sigma \star} v_{C d}^{\Delta} \\
& -v_{C d}^{\Delta \star} \hat{i}_{z}^{\Sigma}+i_{q}^{\Sigma \star}\left(v_{C q}^{\Delta}+v_{C z Q}^{\Delta}\right)-\left(v_{C q}^{\Delta \star}+v_{C z Q}^{\Delta \star}\right) i_{q}^{\Sigma}, \\
y_{5}= & i_{q}^{\Delta \star}\left(\hat{v}_{C z}^{\Sigma}-v_{C d}^{\Sigma}\right)-\left(\hat{v}_{C z}^{\Sigma \star}-v_{C d}^{\Sigma \star}\right) i_{q}^{\Delta}+i_{d}^{\Delta \star} v_{C q}^{\Sigma}-v_{C q}^{\Sigma \star} i_{d}^{\Delta} \\
& +i_{q}^{\Sigma \star}\left(v_{C d}^{\Delta}-v_{C z D}^{\Delta}\right)-\left(v_{C d}^{\Delta \star}-v_{C z D}^{\Delta \star}\right) i_{q}^{\Sigma}+\hat{i}_{z}^{\Sigma \star} v_{C q}^{\Delta} \\
& -v_{C q}^{\Delta \star} i_{z}^{\Sigma}+i_{d}^{\Sigma \star}\left(v_{C z Q}^{\Delta}-v_{C q}^{\Delta}\right)-\left(v_{C z Q}^{\Delta \star}-v_{C q}^{\Delta \star}\right) i_{d}^{\Sigma},
\end{aligned}
$$




$$
\begin{aligned}
y_{6}= & i_{d}^{\Delta \star} v_{C d}^{\Sigma}-v_{C d}^{\Sigma \star} i_{d}^{\Delta}-i_{q}^{\Delta \star} v_{C q}^{\Sigma}-v_{C q}^{\Sigma \star} i_{q}^{\Delta}+i_{d}^{\Sigma \star} v_{C d}^{\Delta}-v_{C d}^{\Delta \star} i_{d}^{\Sigma} \\
& -i_{q}^{\Sigma \star} v_{C q}^{\Delta}-v_{C q}^{\Delta \star} i_{q}^{\Sigma}+\hat{i}_{z}^{\Sigma \star} v_{C z D}^{\Delta}-v_{C z D}^{\Delta \star} \hat{i}_{z}^{\Sigma}, \\
y_{7}= & i_{q}^{\Delta \star} v_{C d}^{\Sigma}-v_{C d}^{\Sigma \star} i_{q}^{\Delta}+i_{d}^{\Delta \star} v_{C q}^{\Sigma}-v_{C q}^{\Sigma \star} i_{d}^{\Delta}+i_{q}^{\Sigma \star} v_{C d}^{\Delta}-v_{C d}^{\Delta \star} i_{q}^{\Sigma} \\
& +i_{d}^{\Sigma \star} v_{C q}^{\Delta}-v_{C q}^{\Delta \star} i_{d}^{\Sigma}+\hat{i}_{z}^{\Sigma \star} v_{C z Q}^{\Delta}-v_{C z Q}^{\Delta \star} \hat{i}_{z}^{\Sigma} .
\end{aligned}
$$

For the overall MT-HVDC system based on MMCs, it suffices to note that the model (III.3) also admits the portHamiltonian representation (IV.1) and the Proposition IV.2 can be applied as well. Interestingly, the same output (IV.4) is obtained for each MMC, which implies that the same PI-PBC controllers ensure global asymptotic stability of the interconnected system in a decentralized fashion.

Remark 4. Notice that the PI-PBC is universal [11], since it can operate in $d c$-voltage control mode, or power control mode, depending on which equilibria are assigned as desired references, and which one is consequently determined by solving (IV.2). As explained in [11], an inherent advantage of this universal property is that there is no need to switch between different controllers when the MMCs are requested to change their mode of operation.

\section{Performance guarantees}

Although stability has been assessed theoretically for the sAAM, no guarantees on the performance of the controller have been provided. Time domain-simulations are then developed both on a single-terminal and a multiterminal HVDC system based on MMCs with the following two objectives. First, to validate the SAAM in port-Hamiltonian form given by (II.9). In fact, recall that this model has been derived under the assumption of negligible high-order harmonics-see Remark 1-an assumption that will be validated for the MMC in single-terminal configuration, when controlled by PI-PBC. ${ }^{3}$ Second, to investigate performances of the PI-PBC for the MMC under single- and multi-terminal configurations that, unfortunately, has been already shown to have serious limitations for the latter when based on 2L-VSCs [11].

For the sake of comparison, we then provide detailed simulations of the following models, which are tested both in single and four-terminal configurations, and are implemented in Matlab/Simulink.

i. The sAAM of a single MMC in port-Hamiltonian form, as expressed by (II.7), and the stationary model of an HVDC system, as expressed by (III.3). Simulation results obtained with these models are identified in the legend of the figures by the corresponding variables name;

ii. The AAM of a single MMC as obtained in Section IIbefore the multi-frequency coordinates transformationand its multiterminal counterpart. This model can be also found in [16], [18], [19] and is implemented using the SimPowerSystem toolbox. Simulation results obtained with this model are identified in the legend of the figures as "AAM".

iii. An Electro-Magnetic Transient (EMT) model of both a single MMC and its multiterminal counterpart, implemented using OPAL-RT Artemis toolbox. The MMC model describes an MMC having 200 sub-modules per arm, with a capacitance of $0.04232 F$ each. This model includes non-linear effects, the switching operations and

\footnotetext{
${ }^{3}$ Similar MMC models have been validated in [13], [14]. However, such models were not described in port-Hamiltonian form and included the wellknown Circulating Current Suppression Control [24].
}

the dynamics of a sub-module arm voltage balancing algorithm. Simulation results with this model are identified in the legend of the figures by "EMT".

For the simulations, we assume that in the multi-terminal configuration all MMCs are characterized by the same parameters and that transmission lines can be represented by the frequency-dependent model detailed in [28], where the line inductance effect is neglected to simplify our tractation ${ }^{4}$. On the other hand, for the single-terminal configuration, we simply consider the case where one of such an MMC is connected to a constant dc current source, rather than the HVDC network. The parameters are summarized in Table I.

Table I: Simulation parameters.

MMC physical and control parameters

\begin{tabular}{rrrrrl} 
& & $R_{f}$ & $0.3429[\Omega]$ & & \\
$S_{n}$ & $1200[\mathrm{MVA}]$ & $L_{f}$ & $62.9[\mathrm{mH}]$ & $K_{P, 1-7}$ & $1 \cdot 10^{-10}$ \\
$U_{n}^{a c}$ & $380[\mathrm{kV}]$ & $N$ & $200[-]$ & $K_{I, 1-4}$ & $1 \cdot 10^{-8}$ \\
$f_{n}$ & $50[\mathrm{~Hz}]$ & $C_{\sigma}$ & $21.16[\mu \mathrm{F}]$ & $K_{I, 5}$ & $2 \cdot 10^{-7}$ \\
$U_{n}^{d c}$ & $620[\mathrm{kV}]$ & $R_{\sigma}$ & $0.6017[\Omega]$ & $K_{I, 6-7}$ & $1 \cdot 10^{-9}$ \\
& & $L_{\sigma}$ & $30.6[\mathrm{mH}]$ & & \\
\hline
\end{tabular}

Transmission line parameters acording to the CIGRE B4 dc grid test system [29]

$0.1906[\mu \mathrm{F} / \mathrm{km}] \quad l^{\prime} \quad 2.112[\mathrm{mH} / \mathrm{km}] \quad r^{\prime} \quad 9.5[\mathrm{~m} \Omega / \mathrm{km}]$

\section{A. Single-terminal configuration}

We consider a single MMC, controlled via a PI-PBC as sketched in Fig. 3, subject to a perturbation applied to the dc current $i_{d c}$, which steps from 0.339 p.u. to 0.423 p.u., at $t=0.05 \mathrm{~s}$. This event automatically demands for a shift of the active current reference $i_{d}^{\Delta \star}$ from $-0.4 p . u$. to -0.5 p.u., to balance the dc current injection. Moreover, we are interested in keeping the reactive power to zero, i.e. $i_{q}^{\Delta \star}=0$, in guaranteeing the objectives R1-R4 as defined in Section II-B, and in obtaining the steady-state the values

$$
m_{z D Q}^{\Delta \star}=\underline{0}_{2}, \quad i_{d q}^{\Sigma \star}=\underline{0}_{2}, \quad v_{C z}^{\Sigma \star}=1.25 .
$$

On the other hand, the remaining references $v_{C d q}^{\Sigma \star}, v_{C d q}^{\Delta \star}$, $v_{C z D Q}^{\Delta \star}$ and $i_{z}^{\Sigma \star}$ can be computed according to the equilibria equations defined by (IV.2), which also determine the steadystate control input $m^{\star}$. All figures involving variables in $d q z$ components are split into two sub-figures showing the $d q$ and the the zero components separately.

The dynamics of the voltage sum and difference are illustrated in Fig. 4 and Fig. 5 respectively. Note that-for clarity of presentation-the latter takes as reference for the zero component the time-periodic signal $v_{C z}^{\Delta}$ rather than its timeinvariant equivalent $v_{z D Q}^{\Delta}$. The following two observations then immediately follow. First, the responses of the sAAM match with great accuracy both the responses of the AAM and the EMT model. Second, the dynamics of the zero component of the voltage sum shows a slow, yet acceptable, convergence rate, which is instead not observed for the zero component of the voltage difference.

The dynamics of the circulating current $i_{d q z}^{\Sigma}$ and grid current $i_{d q}^{\Delta}$ are illustrated in Fig. 6 and Fig. 7 respectively. As for the voltages, responses of the sAAM closely follow the responses of the AAM and EMT model, except for some small, negligible, discrepancy in the latter, which is typically

\footnotetext{
${ }^{4}$ In [28] it was shown that neglecting the inductive effect in transmission lines with only one parallel branch is a good approximation of the frequency dependent model. We adopt this approximation here to prove that the convergence rate of the MT-HVDC is uniquely related to the interconnection pattern and not to the cable states.
} 


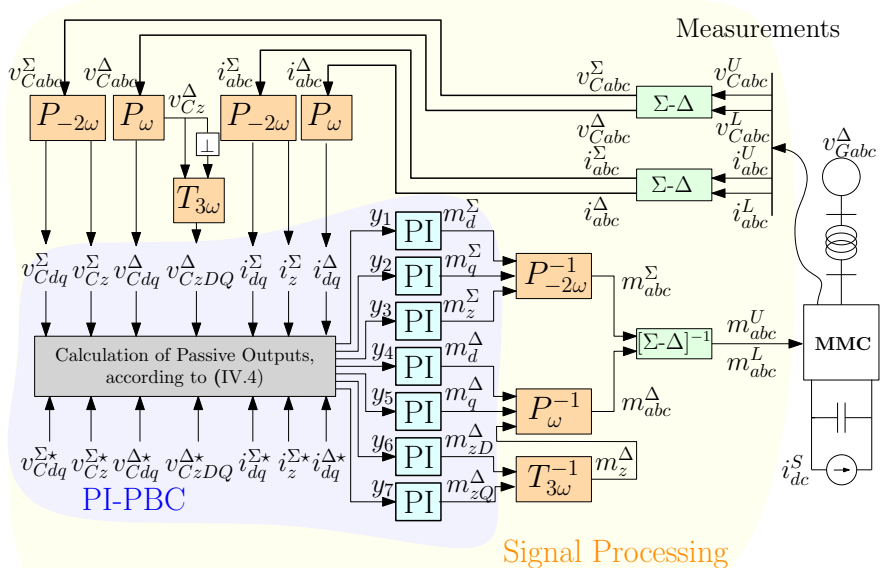

Fig. 3. Control diagram of an MMC in single-terminal HVDC configuration under PI-PBC

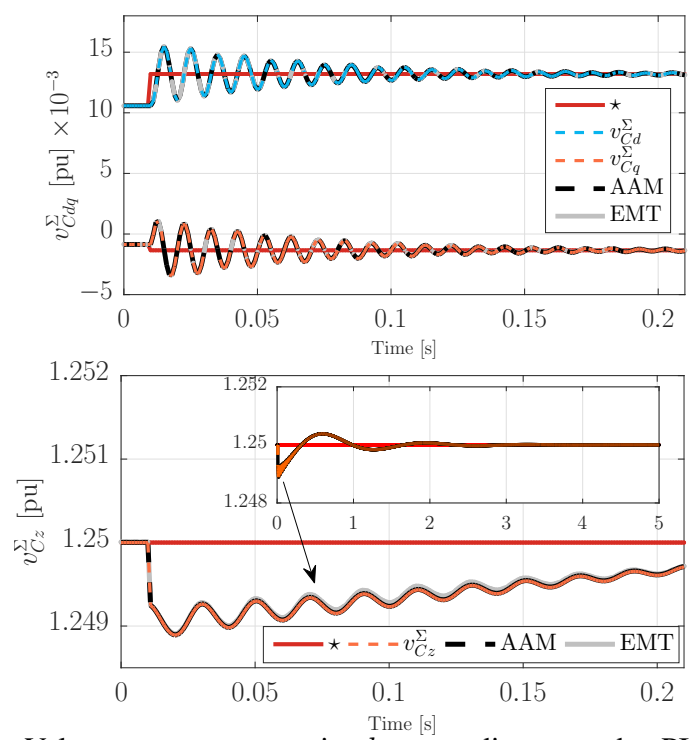

Fig. 4. Voltage sum response in $d q z$ coordinates under PI-PBC in single-terminal configuration.

related to switching dynamics. No significant difference is observed in performance neither for the zero sequence, nor for the direct and quadrature components. Finally, the dynamics of the voltage at the dc terminal $v_{d c}$ is illustrated in Fig. 8. For this variable, a behavior similar to the one observed for the zero component $v_{C z}^{\Sigma}$ is revealed. Nevertheless, despite the slow, yet satisfactory, rate of convergence, the convergence error remains sufficiently small. From these simulations, it can be thus concluded that the SAAM used for the control design describes the system in closed-loop with the PI-PBC with good accuracy.

\section{B. Multi-terminal configuration}

We now consider a four-terminal MT-HVDC system with meshed configuration, as in Fig. 9, where the MMCs are controlled locally via PI-PBC in a decentralized fashion. More precisely, MMC\#1 is operating in dc voltage control mode regulating the dc voltage to $1 p . u$., whereas the rest of the converters are in constant power (current) control mode. The system is perturbed at $t=6 \mathrm{~s}$, by a sudden change in the active current references, which is communicated in a centralized manner by an higher-level controller. More precisely, $i_{d, 2}^{\Delta \star}$ steps down from -0.4 p.u. to -0.5 p.u., $i_{d, 3}^{\Delta \star}$ steps up from 0.6 p.u.
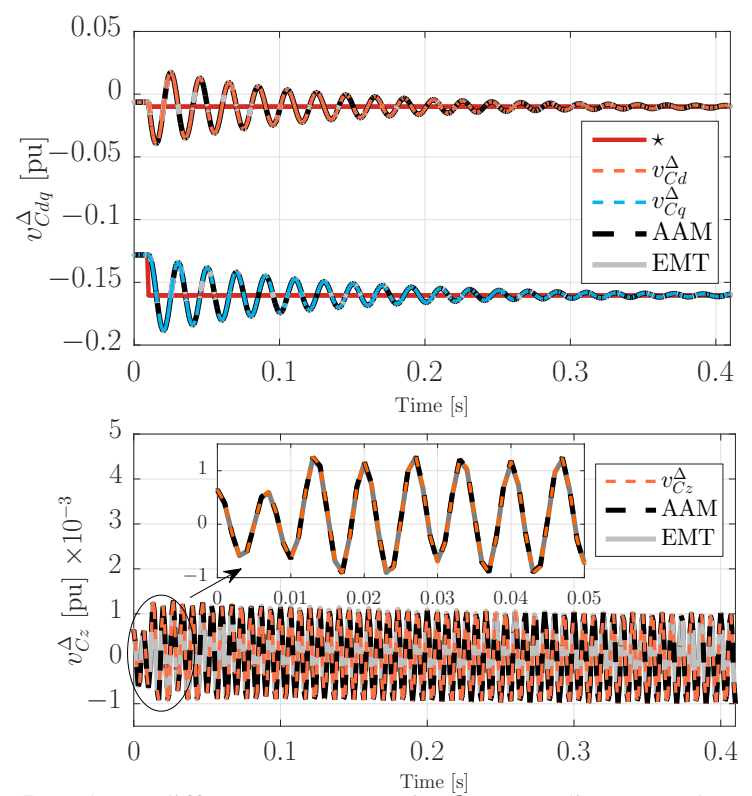

Fig. 5. Voltage difference response in $d q z$ coordinates under PI-PBC in single-terminal configuration.
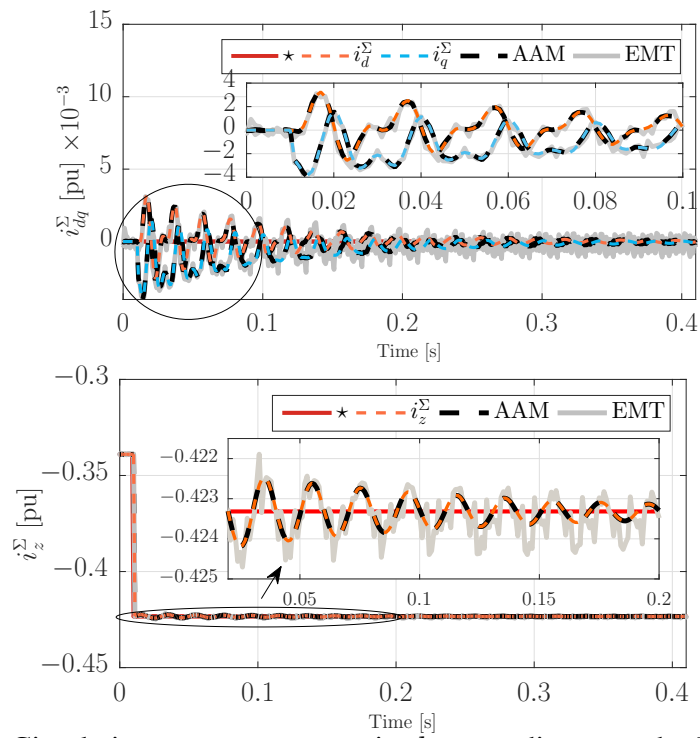

Fig. 6. Circulating current response in $d q z$ coordinates under PI-PBC in single-terminal configuration.

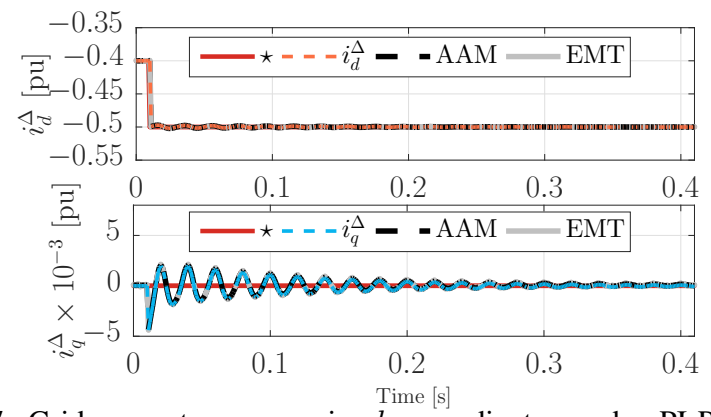

Fig. 7. Grid current response in $d q$ coordinates under PI-PBC in single-terminal configuration.

to 0.7 p.u. and $i_{d, 4}^{\Delta \star}$ steps down from 0.7 p.u. to 0.5 p.u. These simultaneous changes automatically demand for a shift of the active current reference $i_{d, 1}^{\Delta \star}$ such that the voltage $v_{d c, 1}^{\star}$ is kept at its nominal value of 1 p.u.. Moreover, as for the single terminal case, we are interested in keeping the reactive power to zero at each terminal, i.e. $i_{q, i}^{\Delta \star}=0$, and in guaranteeing 


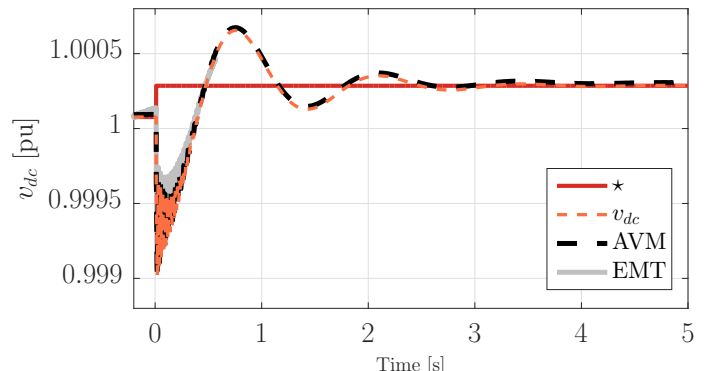

Fig. 8. DC voltage response under PI-PBC in single-terminal configuration.

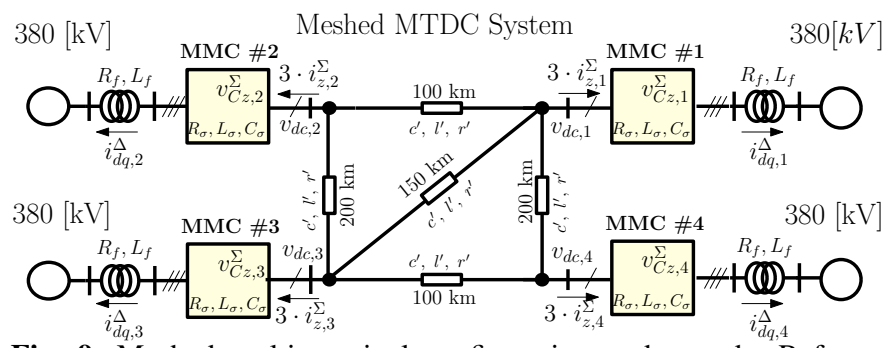

Fig. 9. Meshed multi-terminal configuration under study. Refer to Table I for the MMC and transmission line parameter values.

the requirements R1-R4 as defined in Section II-B, and in obtaining the steady-state values

$$
m_{z D Q, i}^{\Delta \star}=\underline{0}_{2}, \quad i_{d q, i}^{\Sigma \star}=\underline{0}_{2}, \quad v_{C z, i}^{\Sigma \star}=1.25
$$

with $i=1, \ldots, 4$. The remaining references can be thus computed according to the equilibria equations defined by (IV.2), which implicitly define also the steady-state control inputs $m_{i}^{\star}$.

The dc voltage responses $v_{d c, i}$ are illustrated in Fig. 10a, while the zero components of the arm voltage sums $v_{C z, i}^{\Sigma_{\star}}$ are depicted in Fig. 10b. Both dc voltage and zero component variables are characterized by very small steady-state errors. However, in contrast with the results observed for the single-terminal configuration, they show unacceptably slow transients. This is particularly true for the zero components of the voltage sum, which takes approximately two minutes to converge to the steady-state of interest. The responses of the zero component of the circulating currents $i_{z, i}^{\Sigma}$ and grid currents $i_{d, i}^{\Delta}$ are instead illustrated in Fig. 10c. Unlike the voltages, current responses are characterized by good performances.

To illustrate the potential deleterious effects of the aforementioned slow transients, we now consider a perturbation at $t=6$ $s$ where only the voltage sum reference of the first converter $v_{C z, 1}^{\Sigma_{\star}}$ is stepped from 1.25 p.u. to 1.35 p.u.. The dynamics of the MT-HVDC system under this perturbation is illustrated in Fig. 11. The slow convergence rate of $v_{C z, 1}^{\Sigma}$ is shown in Fig. $11 \mathrm{~b}$ where it can be seen that not only convergence requires approximately two minutes, but also the steady-state error is not negligible.

\section{Small Signal Analysis}

Although stability is guaranteed for all positive PI-PBC gains, their choice certainly affects the quality of the transient performance. To evaluate this aspect a small-signal analysis of the closed-loop system is realized-see Fig. 12. First, consider the slowest eigenvalues of the single-terminal configuration, which can be easily identified from Fig. $12 \mathrm{c}$ as the complex conjugated poles $\lambda_{19}, \lambda_{20}$ located at $-2.211 \times 10^{-5} \pm$ 0.0148i. A participation factor analysis [30] is performed for these eigenvalues, revealing that these oscillatory modes are uniquely associated with the controller states $\gamma_{6}$ and $\gamma_{7}$, which are in their turn related with the control inputs $m_{z D}^{\Delta}$ and $m_{z Q}^{\Delta}$-see Fig. 13a. However, no physical states of the converter seem to be affected by this slow oscillatory modes and therefore their effect is not visible in simulations.

For a more accurate analysis, we now consider the participation factor of the second slowest eigenvalue pair $\lambda_{17}-\lambda_{18}$ located at $-0.085 \pm 3.859 i$ and depicted in Fig. 12b, which reveals a strong relationship with the converter states $v_{C z}^{\Sigma}$, as well as with the controller state $\gamma_{3}$ and the dc voltage $v_{d c}$-see Fig. 13b. Moreover, the controller state $\gamma_{3}$ is associated to the MMC input $\hat{m}_{z}^{\Sigma}$. The location of this complex conjugated pole is indeed reflecting the dynamical behaviour observed in $v_{C z}^{\Sigma}$ since the imaginary part corresponds to the visible oscillation of $0.6142 \mathrm{~Hz}$ and attenuation-or neper frequency-of 0.085 $s^{-1}$. Since this last analysis revealed the strong influence of the control state $\gamma_{3}$ in the performance of the system, controller gains associated to $\hat{m}_{z}^{\Sigma}$ are re-tuned in an attempt to improve the settling time. However, as depicted in Fig. $13 \mathrm{c}$, increasing $K_{I}$ has almost no effect in the displacement of the eigenvalues. An interesting aspect is, however, that performance is increased as the absolute value of the active current (power) is increased-see Fig. 13d.

As for the single-terminal case, we perform a small-signal analysis of the closed-loop MT-HVDC system under consideration to prove that the performance and/or slowest convergence rate of the voltage variables cannot be improved by changing the controller gains.

Consider now the slowest eigenvalues of the MT-HVDC system, which can be directly identified in Fig. 12c, located at $-0.00105 \pm 0.0168 i$. A participation analysis is performed for these eigenvalues-see Fig. 14-revealing that these oscillatory modes are local to MMC\#2, and are associated to its controller states $\gamma_{6,2}$ and $\gamma_{7,2}$, which are in turn related with the control inputs $m_{z D, 2}^{\Delta}$ and $m_{z Q}^{\Delta}$. In addition, the participation factor analysis also reveals that these eigenvalues are related to a lesser extent to the physical variables $v_{C z, 2}^{\Sigma}$ and $v_{d c, 2}$. Nonetheless, the influence of this oscillatory mode is rather small on the physical variables extremely slow convergence rate as revealed by the participation factor analysis, as well as by the fact that by removing these poles (selecting $m_{C z D Q}^{\Delta}=\underline{0}_{2}$ ), the convergence rate remains practically unchanged. Shifting our attention to the three following slowest oscillatory modes that are easily identifiable from Fig. 12c and performing a participation factor analysis reveals similar conclusions, yet for the remaining three MMCs, and thus have been omitted for brevity.

For a more accurate analysis, we now consider the participation factor analysis of the three slowest real poles highlighted in Fig. 12c located respectively at $-0.0067,-0.013358$ and -0.0222 . Notice that no equivalent real poles appear in the single-terminal case. The participation analysis results for each of the these three real poles of interest is given in Fig. 15.

The participation factor analysis reveals that these real poles are not local, but instead represent the interactions between the different MMC converter stations. This explains why no equivalent real pole appears in the single-terminal scenario. Moreover, it also explains why the extremely slow behaviour was only observed in the multi-terminal case, whereas the single-terminal presented a faster performance. In all these three real poles of interest, it can be noticed that there is a strong participation of $v_{C z, i}^{\Sigma}$, which is indeed the variable that experiences the slowest convergence rate, as well as a strong participation of the PI-PBC states $\gamma_{3, i}$ associated to the control input $m_{z}^{\Sigma}$, and to a lesser extent a participation of $\gamma_{6}$ 


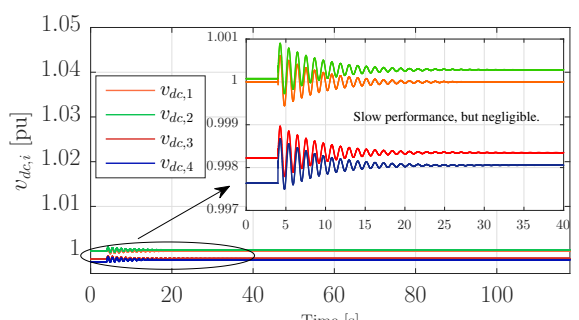

(a)

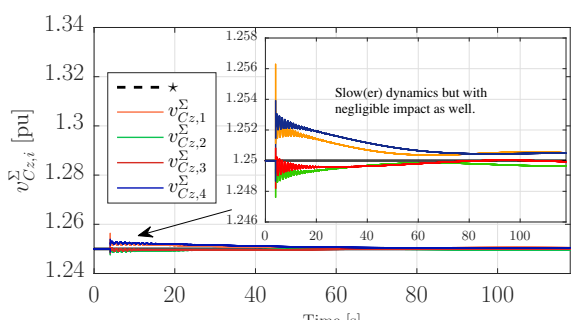

(b)

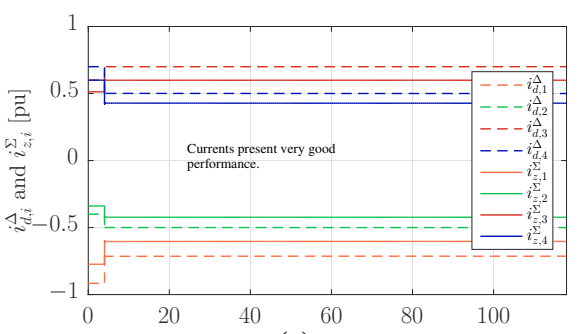

(c)

Fig. 10. Responses in multi-terminal configuration of: (a) dc voltages $v_{d c, i}$; (b) zero component of the voltage sums $v_{C z, i}^{\Sigma}$; (c) zero component of the circulating currents $i_{z, i}^{\Sigma}$ and grid currents $i_{d, i}^{\Delta}$.

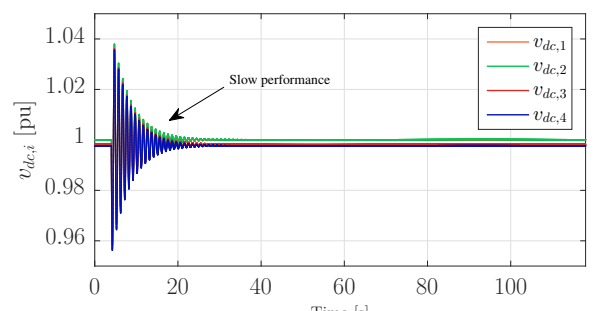

(a)

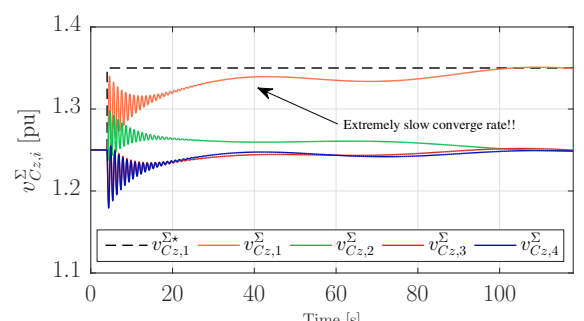

(b)

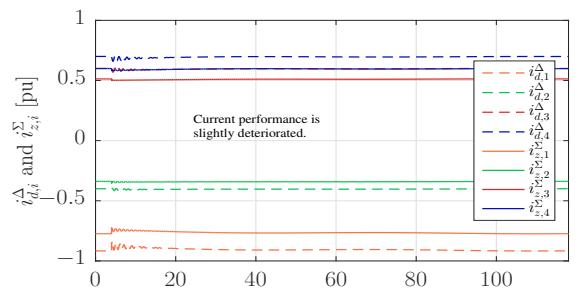

(c)

Fig. 11. Responses in multi-terminal configuration of: (a) dc voltages $v_{d c, i}$; (b) the zero-sequence arm voltage sum $v_{C z, i}^{\Sigma}$, and (c) both circulating $i_{z, i}^{\Sigma}$ and active grid-side currents $i_{d, i}^{\Delta}$ for $i=1 \ldots 4$ in a meshed multi-terminal configuration under a $v_{C z, 1}^{\Sigma}$ reference step.

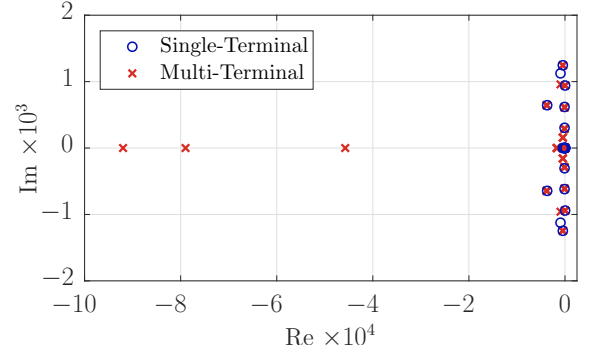

(a)

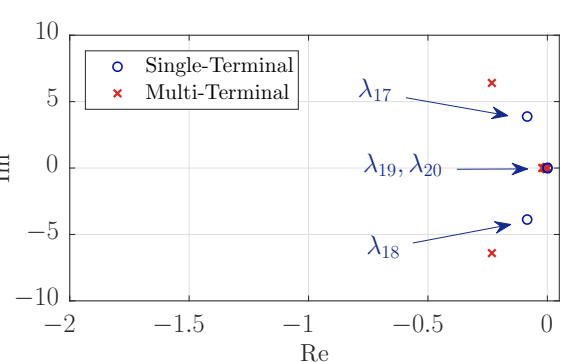

(b)

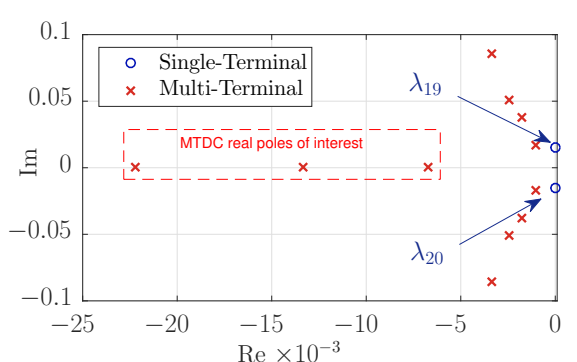

(c)

Fig. 12. Eigenvalues of the MMC(s) in single- and multi-terminal configurations under PI-PBC, and different levels of zoom.

associated to the control input $m_{C z D}^{\Delta}$. Similarly to the singleterminal configuration analysis, the coefficient $K_{I, 3}$ associated to the integral state of the PI-PBC $\gamma_{3}$ is re-tuned two orders of magnitudes without achieving a significant performance improvement, as depicted in Fig. 16.

\section{Outer Loop Control}

It was shown in [31] that it can be advantageous to control the aggregate capacitive energy of the MMC by acting on the circulating current reference for improved performance. Taking inspiration on this approach, we propose to include a conventional outer-loop formed by a simple PI controller acting on the error signal between the zero-sequence arm voltage sum reference $v_{C z}^{\Sigma \text { ref }}$ and its corresponding measured signal $v_{C z}^{\Sigma}$, similar to the one that was used in [31] to improve the stability margins of the $\mathrm{MMC}^{5}$. The output of the PI, defined as $i_{z, i}^{\Sigma *}$ in (V.1) for the $i^{t h} \mathrm{MMC}$, is further added to the desired reference of the circulating current $i_{z}^{\text {rref }}$ to produce the output of the outer loop. However, contrary to the approach in [31] where the output of the outer loop $i_{z}^{\Sigma \text { ref }}$ is implemented by conventional inner current loops, $i_{z}^{\sum *}$ is here added with its desired reference $i_{z, i}^{\sum \mathrm{ref}}$ and is instead assigned to $i_{z}^{\Sigma \star}$, as indicated in the second line of (V.1), and as sketched in Fig. 17. Finally, this new value of $i_{z \star i}^{\Sigma \star}$ will be enforced by the PI-PBC method, through the calculation of the passive output (IV.4).

\footnotetext{
${ }^{5} \mathrm{~A}$ small difference between these outer loops is that the one used in [31] acted on the equivalent energy variable error $\left(w_{C z}^{\Sigma}{ }^{\Sigma}\right.$ ef $\left.-w_{C z}^{\Sigma}\right)$ instead.
}

$$
\begin{aligned}
& i_{z, i}^{\Sigma *}:=-k_{P, i}^{\mathrm{ol}}\left(v_{C z}^{\Sigma \mathrm{ref}}-v_{C z}^{\Sigma}\right)-k_{I, i}^{\mathrm{ol}} \int\left(v_{C z}^{\Sigma \mathrm{ref}}-v_{C z}^{\Sigma}\right) d t \\
& i_{z, i}^{\Sigma \star} \leftarrow i_{z, i}^{\Sigma \mathrm{ref}}+i_{z, i}^{\Sigma *}
\end{aligned}
$$

Replacing (V.1) into the passive output definition (IV.4) will compromise the stability result presented in section IV. More precisely, the stability proof is only valid when $i_{z}^{\Sigma \star}$ is constant and belongs to the admissible equilibrium set $\mathcal{E}$. Therefore, at this point it is necessary to invoke a time-scale separation assumption between the outer-loop and the inner PI-PBC. However, the outer-loop solution has the interesting property of being able to deal with potential instability issues arising in a MTDC grid scenario, by adjusting only the outer loop parameters of the MMCs $k_{P, i}^{\mathrm{ol}}$ and $k_{I, i}^{\mathrm{ol}} 6$.

The time-domain simulation results of the four-terminal MMC-based HVDC are repeated under the same two disturbances as before (step reference change in the active currents $i_{d}^{\Delta}$ and zero-sequence voltage sum $v_{C z}^{\Sigma}$ ) but now with the outer-loop included. The resulting dynamics are depicted respectively in Fig. 18 and Fig. 19. Indeed, the convergence rate of all variables is now very good, particularly for the voltage sum variable (approximately two orders of magnitude faster).

\footnotetext{
${ }^{6}$ Since these are the only parameters that have been excluded in the stability proof, and selecting them both equal to zero would result in the globally asymptotically stable case.
} 


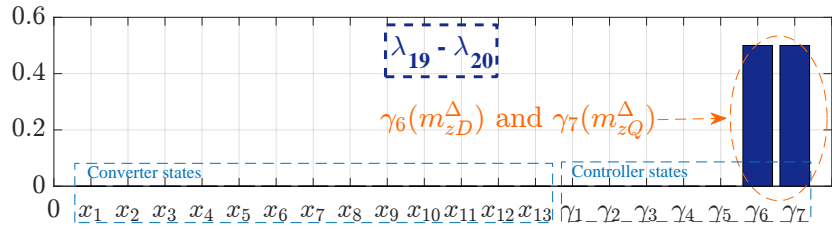

(a)

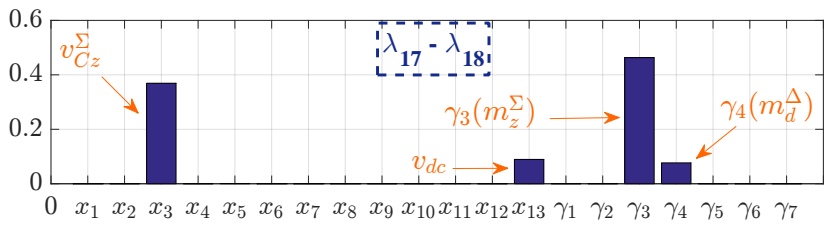

(b)

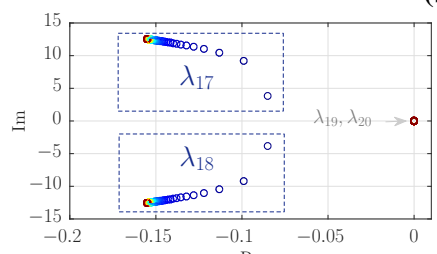

(c)

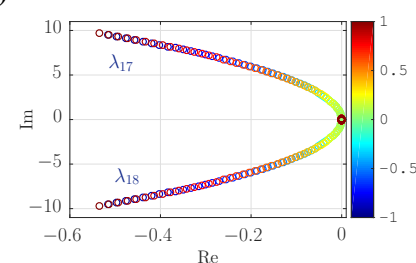

(d)
Fig. 13. Participation factor analysis of: (a) modes $\lambda_{19}-\lambda_{20}$ and (b) $\lambda_{17}-\lambda_{18}$ of the MMC in single-terminal HVDC configuration. Movement of the eigenvalues $\lambda_{17}-\lambda_{18}$ under: (c) a variation of $K_{I, 3}$, from $10^{-8}$ to $10^{-5}$ and (d) a variation of the active current $i_{d}^{\Delta \star}$ from -1 to 1 in p.u..

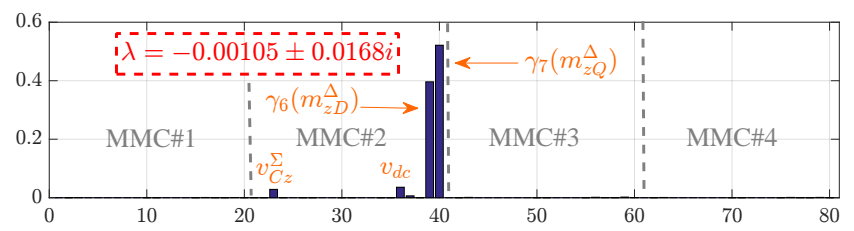

Fig. 14. Participation factor analysis of slowest eigenvalues corresponding to the multi-terminal HVDC configuration.

\section{CONCLUSIONS}

We have applied a PI-PBC to the MMC under both, singleterminal HVDC configuration, as well as for the meshed MTHVDC case.

The procedure takes as a starting point a convenient reformulation of the averaged MMC single-terminal model in $d q z$ coordinates with fixed equilibrium point instead of the standard formulation based on its natural time-periodic coordinates. We then have further manipulated this model such that it can admit a port-Hamiltonian representation suitable for the PI-PBC method, as it allows for the straightforward calculation of the MMC passive output, upon which a standard PI around it renders the system globally stable. In addition, the meshed multi-terminal configuration was also investigated. Interestingly enough, the passive output of the full MT-HVDC system is the union of the passive outputs of each of the individual MMCs, yielding a decentralized controller, while globally stabilizing the interconnected system.

We observed that the controller presents good performance for the single-terminal case, but cannot be further improved by re-tuning of the control parameters, as revealed by a smallsignal eigenvalue analysis. Unfortunately, the situation worsens for the multi-terminal case; i.e., the performance is deteriorated as an unacceptably slow convergence rate dominates the system. A small-signal analysis revealed that the slow performance is related to additional interconnection real poles which only appear in the MT-HVDC case, very close to the origin which cannot be significantly moved via control tuning.

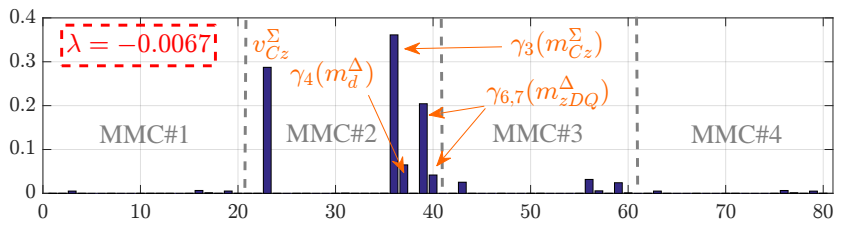

(a)

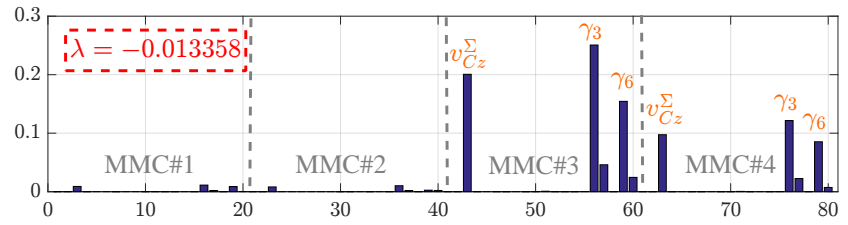

(b)

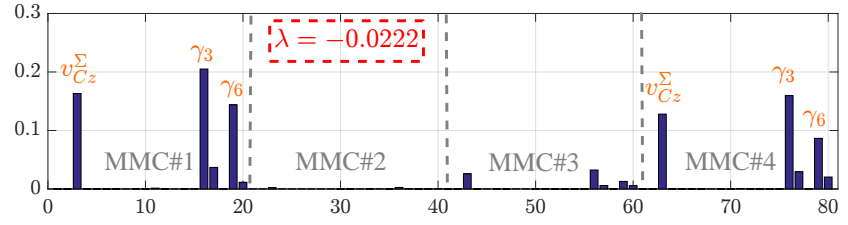

(c)

Fig. 15. Participation factor analysis of the real poles appearing only in the multi-terminal configuration.

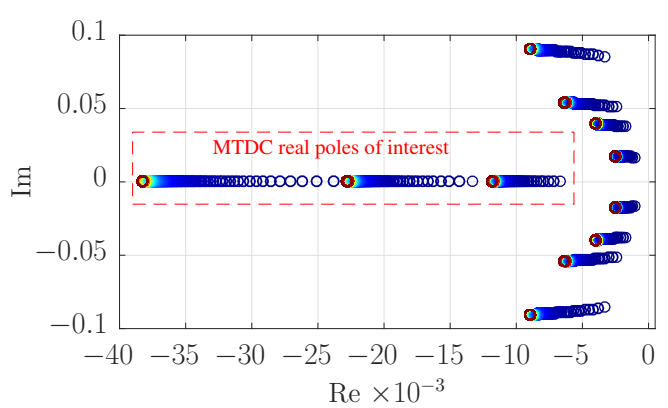

Fig. 16. Movement of eigenvalues of interest corresponding to the multi-terminal configuration, under a variation of $K_{I, 3}$, from $10^{-8}$ to $10^{-6}$.

Finally, we introduced a practical fix in the form of an outerloop control invoking the assumption of time-scale separation with respect to the inner PI-PBC, resulting in improved dynamics for the MT-HVDC configuration.

\section{APPENDIX}

The dissipation matrix $\mathcal{R}$ of the MMC can be expressed as

$$
\mathcal{R}:=\operatorname{diag}\left(\underline{0}_{7}^{\top}, \hat{R}_{\sigma}, \hat{R}_{\sigma}, \hat{R}_{\sigma z}, \hat{R}_{\delta}, \hat{R}_{\delta}, \hat{G}_{d c}\right) \in \mathbb{R}^{13 \times 13}
$$

and the interconnection matrix $\mathcal{J}_{0}$ as:

$$
\mathcal{J}_{0}=\left[\begin{array}{c:c} 
& \\
\overline{\mathcal{J}_{0}} & \frac{\underline{0}_{9}}{4} \\
\hdashline \underline{0}_{9}^{\top}-\overline{4} \underline{\underline{0}}_{2}^{\top} & \underline{0}_{2}
\end{array}\right] \in \mathbb{R}^{13 \times 13}
$$

with $\overline{\mathcal{J}_{0}} \in \mathbb{R}^{12 \times 12}$ defined as

$\overline{\mathcal{J}_{0}}:=\operatorname{bdiag}\left(\mathbb{J}_{2} \hat{C}_{\sigma} 2 \omega, 0, \mathbb{J}_{2} \hat{C}_{\sigma} \omega, \mathbb{J}_{2} \hat{C}_{\sigma} 3 \omega, \mathbb{J}_{2} \hat{L}_{\sigma} 2 \omega, 0, \mathbb{J}_{2} \hat{L}_{\delta} \omega\right)$,

with $\mathbb{J}_{2}:=\left[\begin{array}{rr}0 & 1 \\ -1 & 0\end{array}\right] \in \mathbb{R}^{2 \times 2}$.

Furthermore, the MMC has 7 control modulated interconnection matrices $\mathcal{J}_{h} \in \mathbb{R}^{13 \times 13}$; which can all be directly identified from (II.8), and are given in the following lines. Each of the 7 interconnection matrices can be written as a 


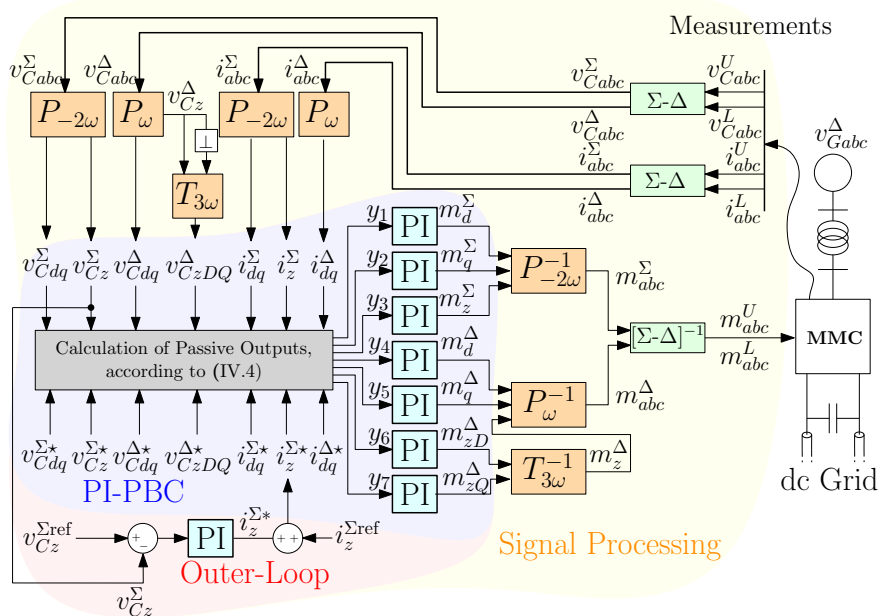

Fig. 17. Control diagram of an MMC in MT-HVDC configuration under PI-PBC $+v_{C z}^{\Sigma}$ outer-loop

function of 4 sub-matrices with the form

$$
\mathcal{J}_{h}:=\left[\begin{array}{c:c}
\overline{\mathcal{J}}_{h} & \underline{0}_{12} \\
-\overline{\mathcal{J}}_{h}^{\top} & \underline{\underline{J}}_{12} \\
\hdashline \underline{0}_{12}^{\top} & 0
\end{array}\right] \in \mathbb{R}^{13 \times 13},
$$

with $\overline{\mathcal{J}_{h}} \in \mathbb{R}^{7 \times 5}$ defined as $\overline{\mathcal{J}_{h}}:=\left[\begin{array}{ll}\overline{A_{h}} & \overline{B_{h}} \\ \overline{C_{h}} & \overline{D_{h}}\end{array}\right]$ for some $\overline{A_{h}} \in \mathbb{R}^{3 \times 3}, \overline{B_{h}} \in \mathbb{R}^{3 \times 2}, \overline{C_{h}} \in \mathbb{R}^{4 \times 3}$ and $\overline{D_{h}} \in \mathbb{R}^{4 \times 2}$. Of these sub-matrices, those which are non-zero are given below for $h=1 \ldots 7$.

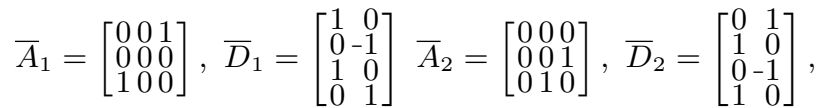

$$
\begin{aligned}
& \bar{A}_{3}=\left[\begin{array}{lll}
1 & 0 & 0 \\
0 & 1 & 0 \\
0 & 0 & \frac{1}{2}
\end{array}\right], \bar{D}_{3}=\left[\begin{array}{ll}
1 & 0 \\
0 & 1 \\
0 & 0 \\
0 & 0
\end{array}\right], \bar{B}_{4}=\left[\begin{array}{ll}
1 & 0 \\
0 & 1 \\
1 & 0
\end{array}\right], \bar{C}_{4}=\left[\begin{array}{lll}
1 & 0 & 1 \\
1 & 0 & 0 \\
1 & 0 & 0 \\
0 & 1 & 0
\end{array}\right],
\end{aligned}
$$

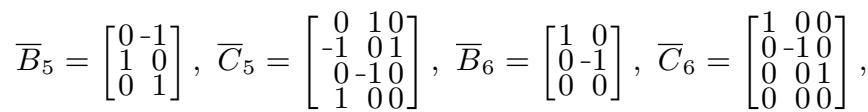

$$
\begin{aligned}
& \bar{B}_{7}=\left[\begin{array}{ll}
0 & 1 \\
1 & 0 \\
0 & 0
\end{array}\right], \bar{C}_{7}=\left[\begin{array}{lll}
0 & 1 & 0 \\
1 & 0 & 0 \\
0 & 0 & 0 \\
0 & 0 & 1
\end{array}\right]
\end{aligned}
$$

Finally, the input and the interconnection vectors of the MMC $E \in \mathbb{R}^{13}$ and $G \in \mathbb{R}^{13}$, can be directly identified from the vector $B$ in (II.7), noting that $B=E+G u$. Thus, for the selected input $u=i_{d c, s}$, they can be respectively defined as

$$
E:=\operatorname{col}\left(\underline{0}_{10},-8 v_{G d q}^{\Delta}, 0\right) ; \quad G:=\operatorname{col}\left(\underline{0}_{12},-\frac{4}{3}\right) .
$$

\section{REFERENCES}

[1] D. V. Hertem, O. Gomis-Bellmunt, and J. Liang, HVDC Grids:For Offshore and Supergrid of the Future. Wiley-IEEE Press Series on Power Engineering, 2016, pp. 528-

[2] A. Lesnicar and R. Marquardt, "An innovative modular multilevel converter topology suitable for a wide power range," in Power Tech Conf. Proc., 2003 IEEE Bologna, vol. 3, June 2003, pp. 6 pp. Vol.3-.

[3] S. Rohner, S. Bernet, M. Hiller, and R. Sommer, "Modulation, losses, and semiconductor requirements of modular multilevel converters," IEEE Trans. on Industrial Electronics, vol. 57, no. 8, pp. 2633-2642, Aug 2010.

[4] “BestPaths EU Project," http://bestpaths-project.eu/, accessed: 2017-0626.

[5] J. Beerten, S. D'Arco, and J. A. Suul, "Identification and small-signal analysis of interaction modes in VSC MTDC systems," IEEE Trans. on Power Delivery, vol. 31, no. 2, pp. 888-897, April 2016.
[6] H. K. Khalil, Nonlinear systems. Prentice hall New Jersey, 1996.

[7] R. Ortega, A. van der Schaft, I. Mareels, and B. Maschke, "Putting energy back in control," IEEE Control Systems, vol. 21, no. 2, pp. 1833, Apr 2001

[8] V. Duindam, A. Macchelli, S. Stramigioli, and H. Bruyninckx, Modeling and control of complex physical systems: the port-Hamiltonian approach. Springer Science \& Business Media, 2009.

[9] A. v. d. Schaft and A. J. Schaft, L2-Gain and Passivity in Nonlinear Control, 2nd ed. Berlin, Heidelberg: Springer-Verlag, 1999.

[10] M. Hernandez-Gomez, R. Ortega, F. Lamnabhi-Lagarrigue, and G. Escobar, "Adaptive PI stabilization of switched power converters," IEEE Trans. on Control Systems Technology, vol. 18, no. 3, pp. 688-698, May 2010

[11] "Modeling and control of HVDC transmission systems from theory to practice and back," Control Engineering Practice, vol. 45, pp. 133 146, 2015.

[12] D. Zonetti, R. Ortega, and A. Benchaib, "A globally asymptotically stable decentralized PI controller for multi-terminal high-voltage dc transmission systems," in 2014 European Control Conf. (ECC), June 2014, pp. 1397-1403.

[13] G. Bergna-Diaz, J. Freytes, X. Guillaud, S. D'Arco, and J. A. Suul, "Generalized voltage-based state-space modelling of modular multilevel converters with constant equilibrium in steady-state," IEEE Journal of Emerging and Selected Topics in Power Electronics, vol. PP, no. 99, pp. 1-1, 2018.

[14] G. Bergna, J. A. Suul, and S. D'Arco, "State-space modelling of modular multilevel converters for constant variables in steady-state," in 2016 IEEE 17th Workshop on Control and Modeling for Power Electronics (COMPEL), June 2016, pp. 1-9.

[15] A. Antonopoulos, L. Angquist, and H. P. Nee, "On dynamics and voltage control of the modular multilevel converter," in 2009 13th European Conf. on Power Electronics and Applications, Sept 2009, pp. $1-10$.

[16] L. Harnefors, A. Antonopoulos, S. Norrga, L. Angquist, and H. P. Nee, "Dynamic analysis of modular multilevel converters," IEEE Trans. on Industrial Electronics, vol. 60, no. 7, pp. 2526-2537, July 2013.

[17] G. Bergna-Diaz, D. Zonetti, S. Sanchez, E. Tedeschi, and R. Ortega, "PI passivity-based control of modular multilevel converters for multiterminal HVDC systems," in 2017 IEEE 18th Workshop on Control and Modeling for Power Electronics (COMPEL), July 2017, pp. 1-8.

[18] S. Rohner, J. Weber, and S. Bernet, "Continuous model of modular multilevel converter with experimental verification," in 2011 IEEE Energy Conversion Congress and Expo., Sept 2011, pp. 4021-4028.

[19] A. Christe and D. Dujić, "State-space modeling of modular multilevel converters including line frequency transformer," in Power Electronics and Applications (EPE'15 ECCE-Europe), 2015 17th European Conf. on, Sept 2015, pp. 1-10.

[20] S. S. Khan and E. Tedeschi, "Modeling of MMC for fast and accurate simulation of electromagnetic transients: A review," Energies, vol. 10, no. 8,2017

[21] H. Saad, S. Dennetiére, J. Mahseredjian, P. Delarue, X. Guillaud, J. Peralta, and S. Nguefeu, "Modular multilevel converter models for electromagnetic transients," IEEE Trans. on Power Delivery, vol. 29, no. 3, pp. 1481-1489, June 2014

[22] _ " "Modular multilevel converter models for electromagnetic transients," IEEE Trans. on Power Delivery, vol. 29, no. 3, pp. 1481-1489, June 2014.

[23] Q. Tu, Z. Xu, and L. Xu, "Reduced switching-frequency modulation and circulating current suppression for modular multilevel converters," IEEE Trans. on Power Delivery, vol. 26, no. 3, pp. 2009-2017, July 2011.

[24] _ "Reduced switching-frequency modulation and circulating current suppression for modular multilevel converters," in Transmission and Distribution Conf. and Expo. (T\&D), 2012 IEEE PES, May 2012, pp. $1-1$.

[25] T. Li, A. M. Gole, and C. Zhao, "Harmonic instability in MMC-HVDC converters resulting from internal dynamics," IEEE Trans. on Power Delivery, vol. 31, no. 4, pp. 1738-1747, Aug 2016.

[26] R. Teodorescu, M. Liserre, and P. Rodríguez, Grid converters for photovoltaic and wind power systems. IEEE/John Wiley \& Sons, 2011, vol. 29.

[27] B. Bollobs, Modern Graph Theory, corrected ed., ser. Graduate texts in mathematics. Heidelberg: Springer, 1998.

[28] J. Beerten, S. D'Arco, and J. A. Suul, "Frequency-dependent cable modelling for small-signal stability analysis of VSC-HVDC systems," 


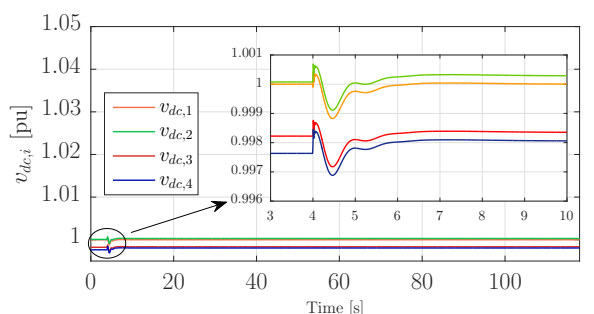

(a)

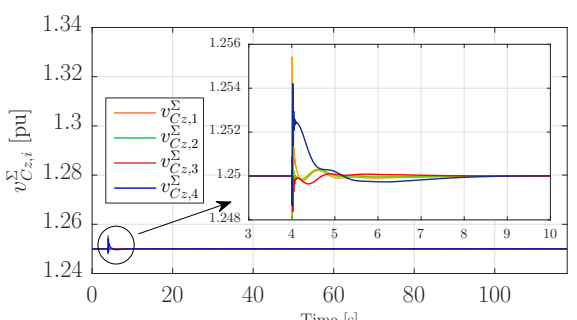

(b)

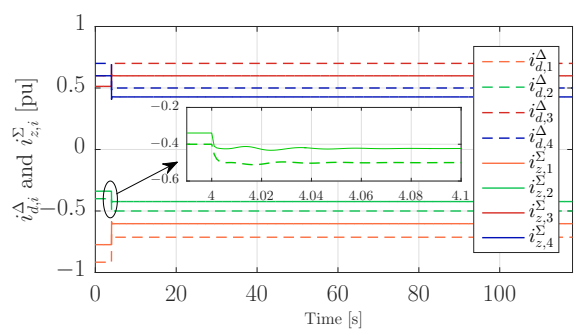

(c)

Fig. 18. PI-PBC $+v_{C z}^{\Sigma}$ outer loop, subject to $i_{d, i}^{\Delta}$ reference changes.

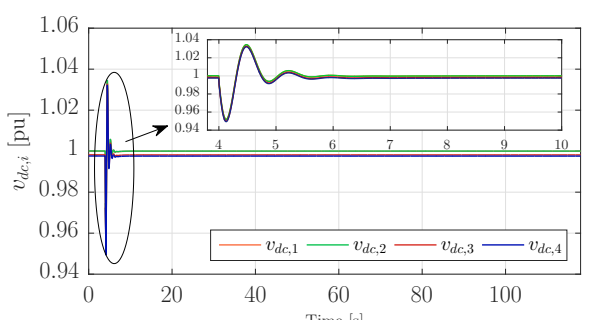

(a)

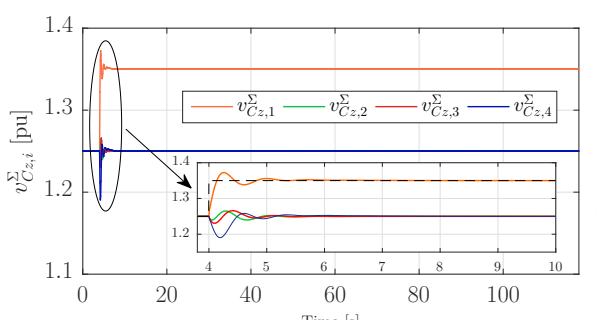

(b)

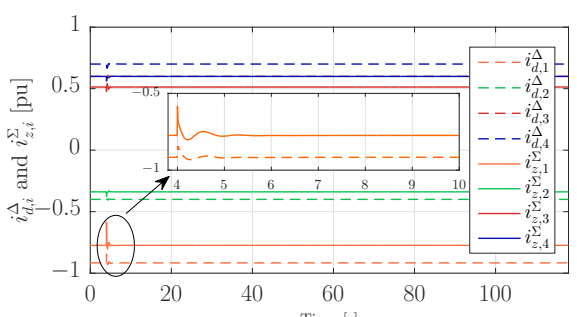

(c)

Fig. 19. PI-PBC $+v_{C z}^{\Sigma}$ outer loop, subject to $v_{C z, 1}^{\Sigma}$ reference changes.

IET Generation, Transmission Distribution, vol. 10, no. 6, pp. 13701381, 2016.

[29] T. Vrana, S. Dennetiere, Y. Yang, J. Jardini, D. Jovcic, and H. Saad, "The Cigré B4 DC grid test system," vol. 270, 102013.

[30] P. Kundur, N. J. Balu, and M. G. Lauby, Power system stability and control. McGraw-hill New York, 1994.

[31] J. Freytes, G. Bergna, J. A. Suul, S. D’Arco, F. Gruson, F. Colas, H. Saad, and X. Guillaud, "Improving small-signal stability of an MMC with CCSC by control of the internally stored energy," IEEE Trans. on Power Delivery, vol. 33, no. 1, pp. 429-439, Feb 2018.

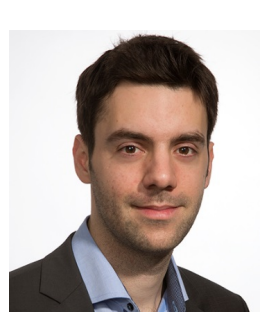

Gilbert Bergna-Diaz received the electrical power engineering degree from the Universidad Simón Bolívar, Caracas, Venezuela, in 2008, the Research Master degree in electrical energy from the École Supérieure d'Électricité (Supélec), Paris, France, in 2010, and the joint Ph.D. degree in electric power engineering from École CentraleSupélec, Paris, and the Norwegian University of Science and Technology (NTNU), Trondheim, Norway, in 2015.

In 2014, he joined SINTEF Energy Research as a Research Scientist, where he was involved in modelling, analysis, and control of HVDC transmission systems. Since 2016, he has been a Post-Doctoral Research Fellow at NTNU, where he is involved in energy-based modelling and nonlinear control of multi-terminal HVDC grids.

Daniele Zonetti received the B.Sc. degree in control engineering from the University of Rome, La Sapienza, Italy, in 2009, and a double Master degree in systems engineering from both La Sapienza and the University of Paris-Sud XI, in 2012. In 2016, he obtained the Ph.D. degree in automatic control from the University of Paris-Saclay. He joined then IMDEA Energy Institute (Madrid, Spain) in 2017, in quality of recipient of a Marie-Curie fellowship. Since 2018, he is a post-doctoral researcher at the Laboratoire de Specification et Verification in Cachan, France. His main research are in first-principles modeling, estimation and nonlinear control of smart energy systems.

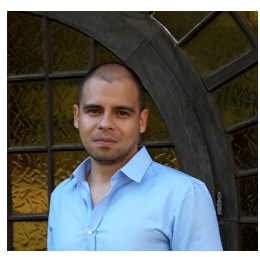

Santiago Sanchez (M'11) received the B.S. and M.Sc. degrees in electrical engineering from the Universidad Tecnologica de Pereira, Pereira, Colombia, in 2006 and 2008, respectively, and the Ph.D. degree from 2015 at the Norwegian University of Science and Technology, Trondheim, Norway.

He is currently a Post-Doctoral Research Fellow at the same university. His research interests include electrical stability in grid dominated by power electronics microgrids and HVDC multiterminal grids.

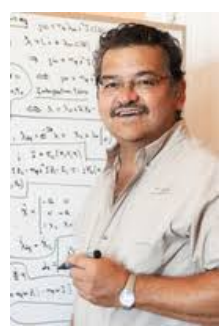

Romeo Ortega was born in Mexico. He obtained his BSc in Electrical and Mechanical Engineering from the National University of Mexico, Master of Engineering from Polytechnical Institute of Leningrad, USSR, and the Docteur D`Etat from the Politechnical Institute of Grenoble, France in 1974, 1978 and 1984 respectively.

He then joined the National University of Mexico, where he worked until 1989. He was a Visiting Professor at the University of Illinois in 1987-88 and at McGill University in 1991-1992, and a Fellow of the Japan Society for Promotion of Science in 1990-1991. He has been a member of the French National Research Council (CNRS) since June 1992. Currently he is a Directeur de Recherche in the Laboratoire de Signaux et Systemes (CentraleSupelec) in Gif-sur-Yvette, France. His research interests are in the fields of nonlinear and adaptive control, with special emphasis on applications.

Dr Ortega has published five books and more than 300 scientific papers in international journals, with an h-index of 81 . He has supervised more than $30 \mathrm{PhD}$ thesis. He is a Fellow Member of the IEEE since 1999 and an IFAC Fellow since 2016. He has served as chairman in several IFAC and IEEE committees and participated in various editorial boards of international journals. He is currently Editor in Chief of Int. J. Adaptive Control and Signal Processing and Senior Editor of Asian Journal of Control.

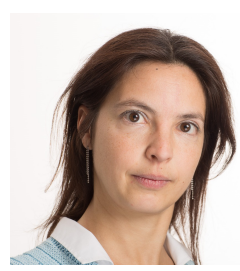

Elisabetta Tedeschi received the M.Sc. degree( with honors) in electrical engineering and the Ph.D. degree in industrial engineering from the University of Padova, Italy, in 2005 and 2009, respectively. From 2009 to 2011, she was a post doc researcher at the Norwegian University of Science and Technology (NTNU), working on the design and control of energy conversion systems for the grid integration of offshore renewable energies. Having received a Marie Curie Fellowship, she was a Researcher at Tecnalia, Spain, from 2011 to 2013, where she worked as a principal investigator in the FP7-Sea2grid Project, related to the storage needs for the grid integration of wave energy converters. From 2013 to 2014, she was Research Scientist at SINTEF Energy and Adjunct Associate Professor at NTNU. In 2014, she became Full Professor within offshore grid at NTNU. She has a core competence in the design and control of energy conversion and transmission systems, with focus on offshore energy, and power-quality issues. She has led and/or contributed to more than 15 national and international scientific projects and she is the author or co-author of 1 book chapter and more than 15 journals and 50 conference papers in the field of marine energy and energy conversion systems. 